IGICS: JGA Keynote Program

The 11th International

Gastrointestinal Consensus

Symposium (IGICS)

\title{
Gastrointestinal Cancer and Gastrointestinal Infection
}

February 10, 2018, Venue Keio Plaza Hotel, Tokyo, Japan

Program \& Abstracts

Guest Editor

Hiroyuki Kato, Tochigi, Japan

\section{Contents}

Program

Oral Session 1

Abstracts IO1-1-|O1-8

Oral Session 2

103

Abstracts IO2-1-102-6

Oral Session 3

Abstracts IO3-1-103-6

Oral Session 4

109

Abstracts IO4-1-IO4-6

112

Abstracts IP1-1-IP1-7

Poster Session 2

Abstracts IP2-1-IP2-7

Author Index for Abstracts 


\section{Outline}

Digestion

IGICS: JGA Keynote Program

The 11th International Gastrointestinal Consensus Symposium (IGICS)

Date February 10 (Sat.), 2018

Venue Keio Plaza Hotel, Tokyo, Japan

\section{IGICS Committee Members}

JGA International Exchange Committee Members as of February 2017

Shin Fukudo, Japan

Ryuichi Iwakiri, Japan

Takeshi Kamiya, Japan

Satoshi Motoya, Japan

Kazunari Murakami, Japan

Akihito Nagahara, Japan

Hidekazu Suzuki, Japan

Toshio Watanabe, Japan

Satoru Yamaguchi, Japan (Secretary General of The 11th IGICS)

IGICS International Active Members

Francis K.L. Chan, Hong Kong, China

Kwong Ming Fock, Singapore

Ki-Baik Hahm, Korea

Udom Kachintorn, Thailand

Abdul Aziz Rani, Indonesia

Jose D. Sollano, Philippines

Qi Zhu, China

\section{KARGER}

(C) 2018 S. Karger AG, Basel 
8:30-9:50 "Hana D", 4th floor, Main Tower, Keio Plaza Hotel

(80min.)

Oral Session 1 "Gastrointestinal Cancer 1 "

Chairpersons: Shin'ichi Takahashi (Kosei Hospital, Japan)

Francis K. L. Chan (The Chinese University of Hong Kong, China)

I01-1 Coproporphyrinogen Oxidase(CPOX) and Protoporphyrinogen Oxidase (PPOX) are Involved in Fluorescence p. 100 Intensity of Laser-Based Photodynamic Endoscopic Diagnosis for Early Gastric Cancer (EGC)

Hiroki Kurumi (Tottori University, Japan)

IO1-2 Molecular Status Changes According to Tumor Location in EGJ Adenocarcinoma

Yu Imamura (The Cancer Institute Hospital of Japanese Foundation of Cancer Research)

IO1-3 Rejuvenating Action of Placenta Derived Mesenchymal Stem Cells Led to Prevention of Helicobacter pyloriAssociated Gastric Cancer

Jong Min Park (CHA University Bundang Medical Center, Korea)

101-4 Automatic Detection of Early Gastric Cancer by Training a Deep Learning Algorithm Wang Dong (Changhai Hospital, China)

I01-5 What Is an Optimal Treatment for Superficial Non-Ampullary Duodenal Epithelial Tumors? Yuichi Kojima (Osaka Medical College, Japan)

IO1-6 Impact of BLI for Detection and Differentiation of superficial GI Neoplasia Tiing Leong Ang (Changi General Hospital, Singapore)

I01-7 Small Bowel Malignancy in Patient with Obscure Gastrointestinal Bleeding in Cipto Mangunkusumo Hospital, Diagnosis by Enteroscopy: A Case Series

Hasan Maulahela (University of Indonesia, Indonesia)

IO1-8 Hepatocellular Carcinoma Surveillance Is No Longer Cost-Effective in Females After Hepatitis B Surface Antigen Seroclearance Younger Than 60 Years Old

Terry Cheuk Fung Yip (The Chinese University of Hong Kong, China)

9:50-10:50 "Hana D", 4th floor, Main Tower, Keio Plaza Hotel (60min.)

\section{Oral Session 2 "Gastrointestinal Cancer 2"}

Chairpersons: Yasuhisa Sakata (Saga Medical School, Japan) Abdul Aziz Rani (University of Indonesia Cipto Mangunkusumo Hospital, Indonesia)

IO2-1 The Utility of the Japanese NBI Expert Team (JNET) versus NICE Classification in the Management of Colorectal Polyps

James Weiquan Li (Changi General Hospital, Singapore)

102-2 Green Tea Extracts for the Prevention of Metachronous Colorectal Polyps Among Patients Who Underwent Endoscopic Removal of Colorectal Adenomas: A Randomized Clinical Trial Dong Ho Lee (Seoul National University Bundang Hospital, Korea)

IO2-3 Fecal Bacteria Act as Novel Biomarkers for Non-Invasive Diagnosis of Colorectal Neoplasm Jessie Qiaoyi Liang (The Chinese University of Hong Kong, China)

102-4 Prediction of the Poor Prognostic Factors for Stage II Colorectal Cancer Yusuke Okuda (Kasugai Municipal Hospital, Japan) 
IO2-5 Emergency Colonic Stenting versus Conventional Open Surgery for Acute Left-Sided Malignant Bowel

Obstruction: A Meta-Analysis

Dennis F. Fernandez (University of Santo Tomas Hospital, Philippines)

IO2-6 Primary Colorectal Lymphomas: A Clinicopathologic Study of 64 Patients Shotaro Nakamura (Iwate Medical University, Japan)

13:10-13:30 “Hana D", 4th floor, Main Tower, Keio Plaza Hotel

(20min.)

IGICS Report on the Result of the Questionnaire

Satoru Yamaguchi (Dokkyo Medical University, Japan)

13:30-14:30 “Hana D", 4th floor, Main Tower, Keio Plaza Hotel

(60min.)

Oral Session 3 "Gastrointestinal Infection 1"

Chairpersons: Kazunari Murakami (Oita University, Japan)

Kwong Ming Fock (Changi General Hospital, Singapore)

IO3-1 Rapid Diagnosis of "Helicobacter pylor" Infection by Gamma-Glutamyltranspeptidase-Activated Fluorescent

p. 106

Probe

Taro Akashi (Nagasaki University Hospital, Japan)

IO3-2 LncRNA THRIL is Involved in the Phosphorylation of cagA and Regulates Helicobacter pylori CagA

p. 106 Induced-Inflammation

Sang Kil Lee (Yonsei University College of Medicine Severance Hospital, Korea)

IO3-3 Walnut Phenolic Extract Alleviates Non-Steroidal Anti-Inflammatory Drug-Induced Gastric Mucosal Damages by Increasing Cyclooxygenase-2 Inhibition, 15-Hydroxyprostaglandin Dehydrogenase Induction, and Heme Oxygenase-1 Induction

Jong-Min Park (CHA Cancer Prevention Research Center, CHA Cancer Institute, CHA University, Korea)

IO3-4 Placenta Derived Mesenchymal Stem Cells Afforded Rejuvenation of Helicobacter pylori-Associated Atrophic Gastritis Better Than Cord Derived or Adipose Tissue Derived MSCs

Jong-Min Park (CHA Cancer Prevention Research center, CHA University, pangyo, Korea)

IO3-5 Microbial Compositional and Ecological Dysbiosis in Gastric Carcinogenesis

Olabisi Oluwabukola Coker (The Chinese University of Hong Kong, China)

IO3-6 Extensive Gastric Mucosal Atrophy May Prevent from Superficial Non-Ampullary Duodenal Epithelial Tumor p. 108 (SNADET)

Yohei Koyama (Tokyo Medical University, Japan)

14:30-15:30 “Hana D”, 4th floor, Main Tower, Keio Plaza Hotel

(60min.)

Oral Session 4 "Gastrointestinal Infection 2"

Chairpersons: Tetsuya Mine (Tokai University, Japan)

Jose D. Sollano (University of Santo Tomas, Manilia, Philippines)

104-1 P-CAB (revaprazan) Prevented Indomethacin-induced Permeability Through Tightening TJs

Young-Min Han (CHA Cancer Prevention Research Center, CHA Cancer Institute, CHA University, Korea)

IO4-2 Verification of Intestinal Microflora-Mediated Mucosal Homeostasis by Immunoglobulin A

Takashi Nagaishi (Tokyo Medical and Dental University, Japan)

104-3 Pathogenic Role of Dysbiosis in the Development of 5-Fluorouracil-Induced Intestinal Mucositis in Mice

Nahla Hamouda (Kyoto Pharmaceutical University, Japan)

IO4-4 Endoscopic Allpication of New Hemostatic Powder (UI-EWD ${ }^{\circledR}$ ) in Gastrointestinal Bleeding p. 110 Yong Woon Shin (Inha University School of Medicine, Korea)

IO4-5 Health-Related Quality of Life and Personal Living Status among Patients with Inflammatory Bowel Disease:

Jun Shen (Shanghai Institute of Digestive Disease, China)

104-6 A Case of Esophageal Motility Disorder in Systemic Sclerosis Treated by Acotiamide

Tsutomu Takeda (Juntendo University Juntendo Tokyo Koto Geriatric Medical Center, Japan) 
15:30-17:10 “Hana D”, 4th floor, Main Tower, Keio Plaza Hotel

(100min.)

IGICS Poster Session 1 \& Poster Session 2

Chairperson: Qi Zhu (Shanghai Jiao Tong University School of Medicine, China)

IP1-1 Clinical Efficacy \& Safety of Mosapride in Functional Dyspepsia Patients

p. 112

Abdul Aziz Rani (Universitas Indonesia, Indonesia)

IP1-2 New Paradigm for Cancer Prevention; Dietary Kimchi Prevents Inflammation and Cancer by Helicobacter

p. 112 pylori in vitro

Yoo Kang (CHA Cancer Prevention Research Center, CHA Cancer Institute, CHA University, Korea)

IP1-3 Walnut Phenolic Extract Inhibited Helicobacter pylori-Induced STAT3 Phosphorylation Through Activation of PPAsRy

Eun-A Kang (CHA Cancer Prevention Research Center, CHA Cancer Institute, CHA University, Korea)

IP1-4 Modified Docetaxel + Cisplatin + Capecitabine (DCX) for Gastric Cancer

Osamu Maeda (Nagoya University Hospital, Japan)

IP1-5 Small Intestinal Lymphoma with Obstructive lleus Requiring Surgery

Saskia Aziza Nursyirwan (Universitas Indonesia, Indonesia)

IP1-6 A Rare Cause of Obscure Occult Gastrointestinal Bleeding by Giardia Lamblia p. 114 Pitulak Aswakul (Samitivej Sukhumvit Hospital, Thailand)

IP1-7 Enteroscopy Findings: Hookworm Infection as Cause of Severe Anemia p. 114 Amanda Pitarini Utari (Universitas Indonesia, Indonesia)

\section{Chairperson: Ki-Baik Hahm (CHA University School of Medicine, Korea)}

IP2-1 Comparison of Mucosal Defect Size and Resection Depth After Hot and Cold Snare Polypectomy Sho Suzuki (Nihon University School of Medicine, Japan)

IP2-2 Linked Color Imaging (LCI) is Clinically Useful for Endoscopic Assessment in Ulcerative Colitis: A Preliminary p. 115 Pilot Study with 10 Patients Kohei Yamanouchi (Takagi Hospital, Japan/Saga Medical School, Japan)

IP2-3 Pretreatment of Probiotic Cocktail Bifico Ameliorates Colitis-Associated Colorectal Cancer in Mice: Transcriptomic Analysis and Gut Flora Profiling Huan Song (Ruijin Hospital, Shanghai Jiaotong University School of Medicine, China)

IP2-4 The Association of Colonic Diverticular Disease and Colonic Adenoma in Filipinos p. 116 Clarel Camille B. Ng (University of Santo Tomas, Manilia, Philippines)

IP2-5 Diverticular Disease of the Colon: An Analysis of the Clinical Characteristics and Risk Factors in Adult Filipino p. 116 Patients

Clarel Camille B. Ng (University of Santo Tomas, Manilia, Philippines)

IP2-6 Retrospective Study of the Preoperative Chemical Disinfection Methods; Olanexidine Gluconate vs.

IP2-7 DNA Methylation Biomarker in Biliary Brush Sample for Diagnosis of Cholangiocarcinoma in Patients with

Varayu Prachayakul (Siriraj Hospital, Mahidol University, Thailand) 


\section{Oral Session 1}

Chairpersons: Shin'ichi Takahashi (Kosei Hospital, Japan) Francis K. L. Chan (The Chinese University of Hong Kong, China)

\section{1-1 \\ Coproporphyrinogen Oxidase (CPOX) and Protoporphyrinogen Oxidase (PPOX) are Involved in Fluorescence Intensity of Laser- Based Photodynamic Endoscopic Diagnosis for Early Gastric Cancer (EGC)}

\author{
Hiroki Kurumi ${ }^{1}$, Kumi Ogiwara², Tsutomu Kanda', \\ Hajime Isomoto ${ }^{1}$ \\ ${ }^{1}$ Medicine and Clinical Science, Faculty of Medicine, \\ Tottori University, Japan; ${ }^{2}$ Department of Gastroenterology \\ and Hepatology, Nagasaki University Hospital, Japan
}

Background and Aim: Laser-based photodynamic endoscopic diagnosis (LPDED) is an optical imaging technology based on the fundamental biological features of porphyrin metabolism in cancer cells. We have found that 5-aminolevulinic acid (5-ALA) can provide a precise detection of early gastric cancer (EGC) during LPDED (Photodiagnosis Photodyn Ther 2015). However, there is an unresolved issue in the differences in fluorescence intensity among histopathological types. This study was conducted to compare clinicopathological features between the LPDED-positive (with red fluorescence) and negative (without red fluorescence) groups, and to examine the expression of coproporphyrinogen oxidase (CPOX) and protoporphyrinogen oxidase (PPOX), primarily involved in porphyrin metabolism.

Methods: Study 1: Thirty-three gastric tumors in 30 patients were assessed by LPDED using a prototype endoscope equipped with a blue laser ray to cause excitation following oral ALA administration. Then, tumors were resected by endoscopic submucosal dissection. CPOX and PPOX expression was examined immunohistochemically in the excised specimens. The tumor immunoreactivity was scored either $0=$ negative; $1=$ moderate, positive with minimal to moderate immunoreactivity; or 2 = strong, strongly positive with intense immunoreactivity after being compared with the corresponding surrounding non-tumor tissue. Study 2: To explore the mechanisms of histopathological diversity in CPOX and PPOX expression of EGC, we performed immunohistochemical analysis using 72 surgically resected specimens of diverse EGC.

Results: Study 1: Among 33 lesions, 26 tumors were detectable by LPDED, whereas 7 were undetectable. Multivariate analysis showed a significant difference in histopathology between the LPDED-positive and negative groups. Expression of CPOX and
PPOX were higher in tub than sig. Study 2: There were significant differences in CPOX and PPOX expression scores among tub, por, and sig.

Conclusion: CPOX and PPOX protein expression could be involved in the fluorescence intensity of LPDED for EGC, possibly reflecting histopathologic features.

\section{1-2 \\ Molecular Status Changes According to Tumor Location in EGJ Adenocarcinoma}

Yu Imamura ${ }^{1,2}$, Shinji Mine1, Naoki Hiki1, Eiji Oki³, Manabu Yamamoto ${ }^{4}$, Masaru Morita ${ }^{4}$, Yoshihiko Maehara ${ }^{3}$, Hideo Baba², Takeshi Sano1, Masayuki Watanabe ${ }^{1}$

${ }^{1}$ Department of Gastroenterological Surgery, the Cancer Institute Hospital of Japanese Foundation of Cancer Research, Tokyo, Japan; ${ }^{2}$ Department of Gastroenterological Surgery, Graduate School of Medical Sciences, Kumamoto University, Kumamoto, Japan; ${ }^{3}$ Department of Surgery and Science, Graduate School of Medical Sciences, Kyushu University, Fukuoka, Japan; ${ }^{4}$ Department of Gastroenterological Surgery, National Hospital Organization, Kyushu Cancer Center, Fukuoka, Japan

Introduction: Although the incidence of esophagogastric junction (EGJ) adenocarcinoma seems to be increasing in Japan, its trend and molecular features are still unclear.

Methods: Using a database $(\mathrm{N}=534)$ from 4 academic institutions in Japan, we investigated 1) recent incidence trends, 2) MSI, and 3) LINE-1 status. 1) annual trends of numbers of surgically resected Siewert type I-III cases were investigated, using 434 cases from three institutions between 2006 and 2015 (completely overlapped across 3 institutions). 2) MSI was examined by fragment analysis using 5 markers (BAT25, BAT26, D2S123, D5S346, and D17S250) in 351 cases. MSI-high was defined as the cases having two or more unstable markers, MSI-low as one unstable marker, and MSS as no unstable marker. 3) LINE-1 methylation analysis was performed by pyrosequencng in 353 cases. Those were categorized into 4 equal groups by quartiles (Q1-4, in ascending order of LINE-1).

Results: 1) The number of patients were gradually increasing, from 33 cases in 2006 to 55 cases in 2015. 2) MSI-high/MSI-low/ MSS were detected in 27 cases $(7.7 \%) / 29$ cases $(8.3 \%) / 295(84 \%)$. Less frequency of MSI-high was observed in esophageal EGJ tumors (MSI-high tumors in Siewert type I/II/III were 0\%/7.9\%/13\%). Five-year relapse-free survival (RFS) of MSI-high (74\%) was better than that of MSS (42.9\%). 3) The overall LINE-1 methylation level $($ mean $\pm \mathrm{SD}$ ) was $65.2 \pm 7.2$. LINE-1 was lower in the tumors at esophageal EGJ (LINE-1 of Siewert type I/II/III, were $65.2 \pm 7.2 / 64.4 \pm$ 
$10.3 / 68.8 \pm 7.9, \mathrm{P}=0.021)$. Lower LINE-1 was associated with worse outcome (5 yr-RFS of Q1/Q2/Q3/Q4 were $41 \% / 35 \% / 54 \% / 62 \%$ ).

Conclusion: The incidence of EGJ adenocarcinoma is gradually increasing in Japan. MSI and LINE-1 status differ according to the tumor location, indicating genetic/epigenetic features of EGJ adenocarcinoma may not be uniform.

\section{1-3 \\ Rejuvenating Action of Placenta Derived Mesenchymal Stem Cells Led to Prevention of Helicobacter pylori-Associated Gastric Cancer}

\author{
Jong Min Park, Young Min Han, Seong Pyo Hong, \\ Weon Jin Ko, Joo Young Cho, Ki Baik Hahm \\ Digestive Disease Center, CHA University Bundang \\ Medical Center, Seongnam, Korea
}

Helicobacter pylori caused gastric cancer via chronic atrophic gastritis (CAG). Therefore, rejuvenation can block the progression of $H$. pylori-associated gastric carcinogenesis, but even the eradication itself did not warrant these progressions. We hypothesized the rejuvenating and regenerating actions of stem cells can afford these benefits. Using human placenta derived mesenchymal stem cells (hPDSCs), we documented the effects of hPDSCs on H. pyloriassociated $\mathrm{CAG}$ in mice. As the objective rejuvenating action, we have measured molecular changes and gastric microbiomes. After 10 times administration of hPDSCs mice were sacrificed 36 weeks $(\mathrm{n}=10)$. Significant changes in mean body weights were noted between control group and hPDSCs treated group $(\mathrm{p}<0.01)$ along with significant changes in pathological scores, especially scores in inflammation, atrophy changes, and even dysplastic changes $(\mathrm{p}<$ 0.01). Lgr5 expressions were significantly increased in hPDSCs treated group along with musashi-1. Inflammatory mediators, MMP, apoptotic markers were all significantly decreased in hPDSC treated group $(\mathrm{p}<0.001)$. Our model that H. pylori-initiated, high salt dietpromoted gastric carcinogenesis model, significant changes in gastric microbiota were noted, but hPDSCs treated group showed changes similar as seen in normal control. hPDSCs or their conditioned media can be considered as rejuvenating agent to revert $H$. pylori-associated premalignant lesions.

\section{1-4 \\ Automatic Detection of Early Gastric Cancer by Training a Deep Learning Algorithm}

\section{Dong Wang \\ Changhai Hospital, China}

Early gastric cancer (EGC) has been paid enough attentions during recent years in China. Although advancement in new endoscopic techniques and extensive training of endoscopists contributed to the diagnosis of EGC, we still suffer great burden on healthcare due to the relatively low detection rate $(<20 \%)$.
Therefore, we focus on a computer-aided method based on deep machine learning for automatic EGC diagnosis that can assist endoscopists in real time to make decision by providing an objective reference during gastroscopy. 5159 images were retrospectively obtained from endoscopic database in our institution, of which 4127 was used for training a deep convolutional neural network (CNN) while 1032 for model validation. The collected endoscopic images were taken under random lighting conditions including white-light, narrow band imaging (NBI), linked color imaging (LCI), blue laser imaging (BLI) and flexible spectral imaging color enhancement (FICE).

At first, lesions in 1002 images of 311 patients who underwent ESD were identified and marked accurately by experienced endoscopists. All of ESD patients were diagnosed with precancerous lesions (HGIN or LGIN) and EGC and confirmed by histology. Then, these neoplastic images were fed into the $\mathrm{CNN}$ along with images of nonneoplastic diseases that used for interference, such as polyps, stromal tumor, and ulcer. The results of our study show that the sensitivity was $93.3 \%$ and the specificity was $85.4 \%$ for specific neoplastic diseases (precancerous lesions and EGC).

In conclusion, machine algorithm based on deep learning had high sensitivity and specificity for detecting EGC. Promising results have shown that the proposed method may contributed to improvement of detection rate, which meets a significant need for diagnosis of EGC, especially in China, the country with huge population but a limited number of experienced endoscopists.

\section{1-5 \\ What Is an Optimal Treatment for Superficial Non-Ampullary Duodenal Epithelial Tumors?}

\author{
Yuichi Kojima', Toshihisa Takeuchi', Yasuhiro Ueda², \\ Masanobu Fukumoto ${ }^{2}$, Haruhiko Ozaki', Satoshi Harada², \\ Kazuhiro Ota ${ }^{2}$, Yoshiko Fujikawa ${ }^{3}$, Kazunari Tominaga ${ }^{3}$, \\ Kazuhide Higuchi ${ }^{2}$ \\ ${ }^{1}$ Endoscopy Center, Osaka Medical College, Japan; \\ ${ }^{2}$ Second Department of Internal Medicine, Osaka Medical \\ College, Japan; ${ }^{3}$ Premier Developmental Research of \\ Medicine, Osaka Medical College, Japan
}

Purpose: Duodenal tumors are rare, and their biological malignancy is unclear. A consensus regarding therapeutic strategies has not been reached. In this study, we present the results of endoscopic treatment and surgery for superficial non-ampullary duodenal epithelial tumors (SNADET) in our hospital, and review residual tumors/ relapse.

Methods: The subjects were 65 patients with SNADET who underwent endoscopic treatment or surgery in our hospital between April 2005 and September 2016. For EMR, strip biopsy with a twochannel scope was selected in all patients.

Results: The median follow-up period was 6 months in 42 patients who underwent EMR, 36.5 months in 8 who underwent ESD and 4 who underwent hybrid ESD, and 10 months in 11 who underwent surgery. The median lesion diameter was $10 \mathrm{~mm}$ in the EMR group, $12.5 \mathrm{~mm}$ in the ESD/hybrid ESD group, and $20 \mathrm{~mm}$ in the surgery group. The rate of patients negative for tumor cells at the margin was $47.6 \%$ in the EMR group, $91.7 \%$ in the ESD/ 
hybrid ESD group and $90.9 \%$ in the surgery group, respectively. Residual tumor cells were detected in 1 (EMR group) (pTis, 1y0, v0, HMX, VMO) of the 65 patients, and additional EMR was performed. Relapse was detected in 1 (EMR group) of the 65 patients, and additional lymph node dissection (pathology:no metastasis) was conducted for non-curative resection (pT1b SM 1,100 $\mu \mathrm{m}, 1 \mathrm{yl}, \mathrm{HMO}, \mathrm{VMO}$ ), but lymph node relapse was noted after 6 months.

Conclusion: The diagnosis and treatment for SNADET is being developing. To improve its effectiveness and safety and minimize invasiveness, the establishment and standardization of treatment methods are required.

\section{1-6 \\ Impact of BLI for Detection and Differentiation of Superficial GI Neoplasia}

Tiing Leong Ang

Department of Gastroenterology and Hepatology, Changi General Hospital, Singapore; Yong Loo Lin School of Medicine, National University of Singapore

The utility of narrow band imaging (NBI) in the diagnosis of superficial GI neoplasia has been long established. Blue laser imaging (BLI) is a relatively more recent image-enhanced endoscopy technique that utilizes a laser light source developed for narrow-band light observation. It has a bright component useful for far view and lesion detection, and a darker mode useful for characterization of lesions during optical magnification. Recent data have shown that it is useful for detection of colonic neoplasia, as well as characterization and differentiation of gastric and colonic neoplasia. In a multicentre RCT, it was shown that compared to white light imaging (WLI), BLI detected a significantly greater mean number of adenoma (WLI $1.01 \pm 1.36$, BLI $1.27 \pm 1.73 ; \mathrm{P}=0.008)$ [1]. Another RCT demonstrated that BLI decreased adenoma miss rate compared to WLI $(1.6 \%$ vs $10 \%, \mathrm{p}=$ $0.001)$ [2]. It has been shown that the VS classification system using NBI for early gastric cancer (EGC) diagnosis can be applied using BLI [3]. A prospective cross over study demonstrated that magnifying BLI was superior to WLI for characterization of EGC [4]. Comparative studies have shown that magnifying BLI had similar utility as NBI for characterization of EGC $[5,6]$. Magnifying BLI has also been shown to be useful for the diagnosis of colorectal polyps [7] and for assessment of the depth of invasion of colorectal neoplasia [8].

\section{References}

1 Ikematsu H, Sakamoto T, Togashi K, et al. Detectability of colorectal neoplastic lesions using a novel endoscopic system with blue laser imaging: a multicenter randomized controlled trial. Gastrointest Endosc. 2017; 86:386-394.

2 Shimoda R, Sakata Y (Fujise T, et al. The adenoma miss rate of blue-laser imaging vs. white-light imaging during colonoscopy: a randomized tandem trial. Endoscopy. 2017;49:186-190.

3 Yoshifuku Y, Sanomura Y, Oka S, et al. Clinical Usefulness of the VS Classifcation System Using Magnifying Endoscopy with Blue Laser Imaging for Early Gastric Cancer. Gastroenterol Res Pract. 2017;2017: 3649705 .
4 Dohi O, Yagi N, Majima A, et al. Diagnostic ability of magnifying endoscopy with blue laser imaging for early gastric cancer: a prospective study. Gastric Cancer. 2017;20:297-303.

5 Kimura-Tsuchiya R, Dohi O, Fujita Y, et al. Magnifying Endoscopy with Blue Laser Imaging Improves the Microstructure Visualization in Early Gastric Cancer: Comparison of Magnifying Endoscopy with NarrowBand Imaging. Gastroenterol Res Pract. 2017;2017:8303046.

6 Dohi O, Yagi N, Yoshida S, et al. Magnifying Blue Laser Imaging versus Magnifying Narrow-Band Imaging for the Diagnosis of Early Gastric Cancer: A Prospective, Multicenter, Comparative Study. Digestion. 2017; 96:127-134.

7 Yoshida N, Yagi N, Inada Y, et al. Ability of a novel blue laser imaging system for the diagnosis of colorectal polyps. Dig Endosc. 2014;26: 250-8.

8 Yoshida N ), Hisabe T, Inada Y, et al. The ability of a novel blue laser imaging system for the diagnosis of invasion depth of colorectal neoplasms. J Gastroenterol. 2014;49:73-80.

\section{1-7 \\ Small Bowel Malignancy in Patient with Obscure Gastrointestinal Bleeding in Cipto Mangunkusumo Hospital, Diagnosis by Enteroscopy: A Case Series}

Hasan Maulahela, Amanda P Utari, Ahmad Fauzi, Aziz Rani

Division of Gastroenterology, Department of Internal Medicine, Medical Faculty University of Indonesia/Cipto

Mangunkusumo National General Hospital, Indonesia

Small bowel malignancy is still a rare case as caused of gastrointestinal bleeding. The symptoms of small bowel malignancy are not specific. One of the symptoms is obscure gastrointestinal bleeding. Obscure gastrointestinal bleeding (OGIB) is defned as recurrent or persistent gastrointestinal bleeding when the result esophagogastroduodenoscopy and colonic endoscopy is negative. OGIB accounts for approximately $5 \%$ of all gastrointestinal bleeding events. Most OGIB events are attributable to small bowel disease. Single and double-balloon enteroscopy, also known as push-and-pull enteroscopy is an endoscopic technique for visualization of the small bowel. Here we present 13 cases of small bowel tumors in patient with obscure gastrointestinal bleeding who underwent single balloon and double entroscopy at Cipto Mangunkusumo Hospital, Jakarta, Indonesia. Most malignancy we found is adenocarcinoma (6 patients), three patients had gastrointestinal stromal tumor (GIST) from the histopathology, while 3 patients were lymphoma and 1 patient with metastatic disease. In this case series we found that the age range (14-55 years old) is younger than the mean age from literatures. This phenomenon was possibly due to earlier exposure of carcinogen (environmental factors) or genetic factors. 


\section{1-8 \\ Hepatocellular Carcinoma Surveillance Is No Longer Cost-Effective in Females After Hepatitis B Surface Antigen Seroclearance Younger Than 60 Years Old}

\author{
Terry Cheuk Fung Yip, Henry Lik Yuen Chan, \\ Vincent Wai Sun Wong, Yee Kit Tse, \\ Francis Ka-Leung Chan, Grace Lai Hung Wong \\ Institute of Digestive Disease, Department of Medicine \\ and Therapeutics, The Chinese University of Hong Kong, \\ Hong Kong, China
}

Introduction: Hepatocellular carcinoma (HCC) may still occur after hepatitis B surface antigen (HBsAg) seroclearance. Recommendation on HCC surveillance after HBsAg seroclearance among different groups of patients is thus necessary. This territorywide study evaluated the impact of age and gender on the risk of HCC development after HBsAg seroclearance and cost-effectiveness of HCC surveillance.

Methods: All chronic hepatitis B patients who had cleared HBsAg from January 2000 to August 2016 were identified from the Hong Kong Hospital Authority electronic health record. Patients with acute hepatitis $\mathrm{B}$, hepatitis $\mathrm{C}$ and $\mathrm{D}$, and prior liver transplantation were excluded. Annual HCC incidence was estimated in each age and gender subgroup, and HCC surveillance was considered to be cost-effective when the incidence exceeded $0.2 \%$ according to the American Association for the Study of Liver Diseases guidelines.

Result: 4,568 patients with HBsAg seroclearance were identifed. At a median (interquartile range) follow-up of 3.4 (1.5-5.0) years, 54 patients developed $\mathrm{HCC}$; the cumulative incidence of $\mathrm{HCC}$ at 1 , 3 and 5 years were $0.9 \%, 1.3 \%$ and $1.5 \%$, respectively. Age above 50 years (adjusted hazard ratio $4.31,95 \%$ confidence interval 1.72 $10.84 ; P=0.002)$ and male gender $(2.47,1.24-4.91 ; P=0.01)$ were two independent risk factors of HCC. Female patients aged $<50$ years $(\mathrm{n}=545)$ had zero risk of HCC in 5 years. Male patients aged $<50$ years $(n=769)$, female patients aged $>50$ years $(n=1,149)$ and male patients aged $>50$ years $(n=2,105)$ had an annual HCC incidence of $0.14 \%, 0.2 \%$ and $0.5 \%$, respectively. Annual HCC incidences were $0.02 \%$ and $0.32 \%$ in female patients aged $<60$ years $(\mathrm{n}=667)$ and $>60$ years $(n=1,027)$, respectively.

Conclusion: HCC surveillance may not be necessary for female patients with HBsAg seroclearance at 60 years or younger, whereas it remains cost-effective in male patients after 50 years and female patients after 60 years at seroclearance.

\section{Oral Session 2}

Chairpersons: Yasuhisa Sakata (Saga Medical School, Japan) Abdul Aziz Rani (University of Indonesia Cipto Mangunkusumo Hospital, Indonesia)

\section{2-1 \\ The Utility of the Japanese NBI Expert Team (JNET) versus NICE Classification in the Management of Colorectal Polyps}

\author{
James Weiquan Li, Kwong Ming Fock \\ Department of Gastroenterology and Hepatology, Changi \\ General Hospital, Singapore
}

Introduction: Colonoscopy for screening and surveillance reduces colorectal cancer mortality. The JNET classification, unlike NICE classification, differentiates high grade intramucosal neoplasia (HGIN) and superficial submucosal invasive cancer from low grade intramucosal neoplasia (LGIN) as Type 2B. However, JNET is less widely used outside of Japan. The aim of this study is to evaluate the utility of the JNET and NICE classifications when applied to real-life cases in a tertiary centre in Singapore.

Methods: Retrospective study of patients undergoing colonoscopy in the Changi General Hospital between December 2014 and June 2017. Detailed photographs of all colorectal polyps were digitally stored. Histology of resected specimens was recorded. 26 cases were selected from this database, representing a mix of hyperplastic polyp, sessile serrated adenoma, LGIN, HGIN, superficial submucosal invasive cancer and deep submucosal invasive cancer. Two blinded Gastroenterologists (G1 and G2) were asked to classify the polyps according to Paris, JNET and NICE classifications. This was correlated with histology. Interobserver agreement and accuracy of the individual Gastroenterologists were calculated.

Result: Median age of patients 68.5 years, $42.6 \%$ (12/26) male, and average size of colorectal polyps $23 \mathrm{~mm}$. The JNET classification had a superior interobserver agreement compared to the NICE classification, with kappa values of 0.52 and 0.32 respectively. However, G1 and G2 were able to more accurately classify polyps into their matching histology using the NICE classification than the JNET classification (correct responses $69.2 \%$ vs $46.2 \%$ and $73.1 \%$ vs $38.5 \%$ respectively). When only patients with HGIN or superficial submucosal invasive cancer were analyzed, G1 and G2 were able to classify the polyps correctly into Type $2 \mathrm{~B}$ using the JNET classification in $42.9 \%(3 / 7)$ and $57.1 \%(4 / 7)$ of cases respectively.

Conclusion: JNET classification has better interobserver agreement than NICE, and can be used to differentiate HGIN and superficial submucosal invasive cancer from LGIN. However, more training is required to improve its accuracy in a real-world setting. 


\section{2-2 \\ Green Tea Extracts for the Prevention of Metachronous Colorectal Polyps Among Patients Who Underwent Endoscopic Removal of Colorectal Adenomas: A Randomized Clinical Trial}

\section{C.M. Shin, D.H. Lee, A.Y. Seo, H.J. Lee, S.B. Kim, W.C. Son, Y.K. Kim, S.J. Lee, S.H. Park, N. Kim, Y.S. Park, H. Yoon \\ Divison of Gastroenterology, Department of Internal Medicine, Seoul National University Bundang Hospital, Seongnam-si, Gyeonggi, Gyeonggi-do, Korea}

Objectives: To determine the preventive effect of green tea extract (GTE) supplements on metachronous colorectal adenoma and cancer in the Korean population.

Materials and Methods: One hundred seventy-six subjects ( 88 per each group) who had undergone complete removal of colorectal adenomas by endoscopic polypectomy were enrolled. They were randomized into 2 groups:supplementation group ( 0.9 g GTE per day for 12 months) or control group without GTE supplementation. The 72-h recall method was used to collect data on food items consumed by participants at baseline and the 1-year follow-up during the past $48 \mathrm{~h}$. Follow-up colonoscopy was conducted 12 months later in 143 patients (71 in control group and 72 in the GTE group.

Results: Of the 143 patients completed in the study, the incidences of metachronous adenomas at the end-point colonoscopy were $42.3 \%$ (30 of 71) in control group and 23.6\% (17 of 72) in GTE group (relative risk [RR], 0.56; 95\% confidence interval [CI], 0.34-0.92). The number of relapsed adenoma was also decreased in the GTE group than in the control group $(0.7 \pm 1.1$ vs. $0.3 \pm 0.6, p=0.010)$. However, there were no significant differences between the 2 groups in terms of body mass index, dietary intakes, serum lipid profiles, fasting serum glucose, and serum C-reactive protein levels (all $\mathrm{p}>$ $0.05)$.

Conclusion: This study of GTE supplement suggests a favorable outcome for the chemoprevention of metachronous colorectal adenomas in Korean patients (ClinicalTrials.gov number, NCT02321969).

\section{2-3 \\ Fecal Bacteria Act as Novel Biomarkers for Non-Invasive Diagnosis of Colorectal Neoplasm}

Jessie Qiaoyi Liang, Francis K.L. Chan, Joseph J.Y. Sung, Jun Yu

Institute of Digestive Disease and Department of Medicine and Therapeutics, State Key Laboratory of Digestive Disease, Li Ka Shing Institute of Health Sciences, CUHK Shenzhen Research Institute, The Chinese University of Hong Kong, Hong Kong, China

Introduction: Gut microbiota have been implicated in the development of colorectal cancer (CRC). We evaluated the utility of fecal bacterial marker candidates identified by our metagenome sequencing analysis for diagnosis of colorectal neoplasia.
Methods: 557 subjects (203 CRC, 118 advanced adenomas and 236 healthy subjects) from two independent Asian cohorts were included. Probe-based duplex quantitative PCR (qPCR) assays were established for quantification of bacterial marker candidates.

Result: Candidates identified by metagenome sequencing, including Fusobacterium nucleatum ( $F n$ ), Bacteroides clarus (Bc), Roseburia intestinalis Ri Clostridium hathewayi (Ch), and one undefined species (labeled as $m 7$ ), were examined in fecal samples of 203 CRC patients and 236 healthy controls by duplex-qPCR. Strong positive correlations were demonstrated between the quantification of each candidate by our qPCR assays and metagenomics approach $(\mathrm{r}=0.801-0.934$, all $\mathrm{P}<0.0001)$. $F n$ was significantly more abundant in CRC than controls $(\mathrm{P}<0.0001)$, with area under receiver operating curve (AUROC of $0.868(\mathrm{P}<0.0001)$. At the best cutof value maximizing sum of sensitivity and specificity, $F n$ discriminated CRC from controls with a sensitivity of $77.7 \%$, and specificity of $79.5 \%$ in cohort I. A simple linear combination of four bacteria $(F n+C h+\mathrm{m} 7-B c)$ showed an improved diagnostic ability compared to $F n$ alone $($ AUROC $=0.886, \mathrm{P}<0.0001)$ in cohort I. These findings were further confirmed in an independent cohort II. In particular, improved diagnostic performances of $F n$ alone (sensitivity $92.8 \%$, specificity $79.8 \%$ ) and four-bacteria (sensitivity $92.8 \%$, specificity $81.5 \%$ ) were achieved in combination with fecal immunochemical test (FIT) for the detection of CRC. The feasibility of four-bacteria in detecting advanced precancerous neoplasms was further evaluated. Results showed that the four-bacteria detected $36.4 \%$ of advanced adenomas, significantly more sensitive than FIT $(13.6 \%$; P $<0.0001)$. Further combination of four-bacteria and FIT showed an improved detection rate for advanced adenomas of up to $44.1 \%$.

Conclusion: Stool-based CRC-associated bacteria can serve as novel non-invasive diagnostic biomarkers for colorectal neoplasia. This study identified a valuable panel of bacterial markers (F. nucleatum, B clarus, Chathewayi and one undefined species) to improve the diagnostic performance of FIT for colorectal cancers and advanced adenomas.

\section{2-4 \\ Prediction of the Poor Prognostic Factors for Stage II Colorectal Cancer}

\author{
Yusuke Okuda1,3, Tomonori Yamada², Takaya Shimura3, \\ Yoskazu Hirata1, Ryuzo Yamaguchi', Eiji Sakamoto, \\ Satoshi Sofue1, Takahiro Nakazawa², Takashi Joh ${ }^{3}$ \\ ${ }^{1}$ Department of Gastroenterology, Kasugai Municipal \\ Hospital, Japan; 'Department of Gastroenterology, \\ Japanese Red Cross Nagoya Daini Hospital, Japan; \\ ${ }^{3}$ Department of Gastroenterology and Metabolism, \\ Nagoya City University Graduate School of Medical \\ Sciences, Japan; “'Department of Surgery, Kasugai \\ Municipal Hospital, Japan; ${ }^{5}$ Department of Surgery, \\ Japanese Red Cross Nagoya Daini Hospital, Japan
}

Background: Adjuvant therapy should not be routinely recommended for stage II colorectal cancer (CRC) patients. Patients with poor prognostic factors may be treated with postoperative chemotherapy, however, these factors have not been identified.

\section{4}

Digestion 2019;99:95-119

DOI: $10.1159 / 000494404$
The 11th International Gastrointestinal Consensus Symposium (IGICS) 
Methods: Data were retrospectively reviewed from patients with stage II CRC who underwent surgery between January 2007 and December 2011 at two Japanese institutions. We analyzed overall survival (OS) and relapse-free survival (RFS), according to various prognostic factors.

Results: In total, 497 patients with stage II CRC were identified for this study. Five-year OS and RFS for stage II CRC were $84.5 \%$ and $76.0 \%$.

On the univariate analysis, T4 stage $(\mathrm{P}>0.001)$, colorectal obstruction $(\mathrm{P}=0.002)$, poorly differentiated carcinoma $(\mathrm{P}=0.009)$, adjuvant chemotherapy $(\mathrm{P}=0.023)$, the number of lymph node dissected $>12$ $(\mathrm{P}=0.022)$ and age $\geq 70(\mathrm{P}=0.001)$ were significantly poor prognostic factors for OS, and T4 stage $(\mathrm{P}>0.001)$ and colorectal obstruction $(\mathrm{P}=0.008)$ were significantly poor prognostic factors for RFS.

On the multivariate analysis, T4 stage (hazard ratio (HR) 3.521 (95\% CI, 2.132-5.814), P > 0.001), colorectal obstruction (HR 2.646 (95\% CI, 1.462-4.785), $\mathrm{P}=0.001)$, poorly differentiated carcinoma (HR $2.770(95 \% \mathrm{CI}, 1.359-5.650), \mathrm{P}=0.005)$ and age $\geq 70$ (HR 2.137 (95\% CI, 1.300-3.521), $\mathrm{P}=0.003$ ) were significantly poor prognostic factors for OS, and T4 stage (HR 2.941 (95\% CI, 1.927-4.484), P > 0.001 ) and colorectal obstruction (HR 2.146 (95\% CI, 1.266-3.636), $\mathrm{P}=0.005)$ were significantly poor prognostic factors for RFS.

Conclusions: Colorectal obstruction and T4 stage are independent poor prognostic and relapse factors for stage II CRC. Adjuvant chemotherapy might be feasible for stage II CRC with colorectal obstruction and T4 stage.

\section{2-5 \\ Emergency Colonic Stenting versus Conventional Open Surgery for Acute Left-Sided Malignant Bowel Obstruction: A Meta-Analysis}

\section{Dennis F. Fernandez, Timothy Bren Phoa, Clare/ B. Ng \\ Department of Internal Medicine, Section of \\ Gastroenterology, University of Santo Tomas Hospital, Manila, Philippines}

Introduction: Colorectal carcinoma can present with acute intestinal obstruction in $7 \%$ to $30 \%$ of cases, especially if tumor is located at or distal to the splenic flexure. In these cases, emergency surgical decompression becomes mandatory as traditional treatment option. A self-expanding metallic stent (SEMS) is an intraluminal device deployed endoscopically at the point of obstruction and slowly expand radially to their maximum diameter thereby achieving patency of the obstructed anatomy. SEMS provides relief of obstruction, provides time for ample preparation for surgery and palliation in poor surgical candidates. This study attempts to determine the effectiveness and safety of colonic stenting with the use of self-expanding metallic stents versus conventional open surgery in patients presenting with acute left bowel obstruction secondary to malignancy.

Methods: Employing meta-analysis design, studies on selfexpanding metallic stents (SEMs) and left-colonic obstruction were searched in PubMed, ScienceDirect, EBSCO, ProQuest, and Cochrane Library using Boolean logic, keywords, and controlled vocabulary. Two independent reviewers evaluated the identified articles using the P.I.C.O.T. Framework with the following outcomes: stoma rates, mortality, morbidity, and overall survival rate. Using STATA, subgroup analysis and metaregression were employed to account for significant heterogeneity while Egger's test evaluated publication bias.

Result: Twenty clinical trials were reviewed. Pooled estimates using Mantel-Haenzel technique and random-effects model showed lower risk for stomata rates $(R R=0.35, \mathrm{p}<0.01)$, mortality $(R R=$ $0.55, \mathrm{p}=0.0001)$, and short-term morbidity $(\mathrm{RR}=0.42, \mathrm{p}<0.01)$. Risk for long-term complications $(\mathrm{RR}=1.04, \mathrm{p}=0.92)$ and overall survival $(1 \mathrm{HR}=1.20, \mathrm{p}=0.21)$ were not different between SEMs and non-SEMs. Funnel plots analysis and Egger's test suggest/does not suggest publication bias.

Conclusion: SEMS appears to be an effective treatment of acute left-sided malignant large bowel obstruction, as it provides less number of stoma rates, lower mortality rates, and lower incidence of acute complications, with no significant difference in overall survival compared to conventional open surgery.

\section{2-6 \\ Primary Colorectal Lymphomas: A Clinicopathologic Study of 64 Patients}

Shotaro Nakamura ${ }^{1}$, Shunichi Yanai1, Keisuke Kawasaki1, Yuichi Matsuno², Shinichi Kawano², Takehiro Torisu², Minako Fujiwara ${ }^{3}$, Motohiro Esaki², Takayuki Matsumoto ${ }^{1}$

${ }^{1}$ Division of Gastroenterology, Department of Internal Medicine, Iwate Medical University, ${ }^{2}$ Department of Medicine and Clinical Science, Graduate School of Medical Sciences, Kyushu University, ${ }^{3}$ Department of Anatomic Pathology, Graduate School o Medical Sciences, Kyushu University

Introduction: Primary colorectal lymphoma (PCL) is rare, accounting for $10-20 \%$ of gastrointestinal lymphomas, and only for $0.2-0.6 \%$ of all large bowel malignancies.

Methods: We retrospectively analyzed the clinicopathologic characteristics of 64 patients with PCL.

Results: The most frequent involved site was the cecum (52\%), followed by the rectum (40\%). Histologically, mucosa-associated lymphoid tissue (MALT) lymphoma was the most common (38\%), followed by difuse large B-cell lymphoma (DLBCL; 28\%). Treatment modalities included the surgical resection $(n=22)$, chemotherapy $(\mathrm{n}=18)$, resection plus chemotherapy $(\mathrm{n}=12)$, antibiotics such as Helicobacter pylori eradication $(\mathrm{n}=6)$, radiotherapy $(\mathrm{n}=3)$, and watch-and-wait strategy $(\mathrm{n}=3)$. As a result, complete remission $(\mathrm{CR})$ of lymphoma was achieved in 41 patients $(64 \%)$. During the followup period ranging from 0.1 to 22 (median, 4) years, 19 patients $(30 \%)$ showed relapse or progression of lymphomas, and 27 patients $(42 \%)$ died of various diseases. The probabilities for overall survival (OS) and progression-free survival (PFS) 5 years after the diagnosis were $59 \%$ and $49 \%$, respectively. Multivariate analyses using Cox proportional hazard model identified indolent histology (MALT or follicular lymphomas) and early stage ( $\mathrm{I}$ or $\mathrm{II}_{1}$ ) as the independent favorable prognostic factors for OS, whilst B-cell phenotype and early stage for PFS.

Conclusion: Therapeutic strategy for PCLs should be tailored based on the histology and clinical stage. Surgical resection is the treatment of choice for localized tumors. 


\section{Oral Session 3}

\section{Chairpersons: Kazunari Murakami (Oita University, Japan) Kwong Ming Fock (Changi General Hospital, Singapore)}

\section{3-1 \\ Rapid Diagnosis of Helicobacter pylor Infection by Gamma- Glutamyltranspeptidase-Activated Fluorescent Probe}

\author{
Taro Akashi, Kayoko Matsushima', Tsutomu Kanda², \\ Masayuki Nakano ${ }^{3,4}$, Mako Kamiya ${ }^{5}$, Yuko Akazawa ${ }^{1}$ \\ Ken Ohnita', Fuminao Takeshima', Kazuhiko Nakao', \\ Yasuteru Urano ${ }^{5}$, Hajime /somoto $^{2}$ \\ ${ }^{1}$ Department of Gastroenterology and Hepatology, \\ Nagasaki University Hospital, Japan; ${ }^{2}$ Division of \\ Medicine and Clinical Science, Tottori University Hospital, \\ Japan; ${ }^{3}$ Department of Bacteriology, Institute of Tropical \\ Medicine, Nagasaki University, Japan; ${ }^{4}$ Department \\ of International Health, Institute of Tropical Medicine, \\ Nagasaki University, Japan; ${ }^{5}$ Laboratory of Chemical \\ Biology and Molecular Imaging, Graduate School of \\ Medicine, The University of Tokyo, Japan
}

Introduction: gamma-glutamyltranspeptidase (GGT) is a cell surface-associated enzyme that is not highly expressed in normal cell. However GGT is overexpressed in several type of human cancers. It is known that Helicobacter pylori (H. pylori) also produce GGT. Urano et al developed an enzymatically activatable fluorescent probe, gamma-glutamyl hydroxymethyl rthodamine green (gGlu-HMRG), which is nonfluorescent under normal cellular environment, but turns to be highly fluorescent upon reaction with GGT. Accordingly, the aim of this study is to investigate whether gGlu-HMRG can be useful for rapid diagnosing infection $H$. pylori.

Methods: In this study, we investigated whether activation of gGlu-HMRG fluorescence detects a wild-type of $H$. pylori (WT) and a ggt gene-disrupted mutant of $H$. pylori (ggt mutant). In addition, we investigated whether activation of gGlu-HMRG fluorescence was suppressed in $H$. pylori culture solution which was co-incubated with an inhibitor of GGT (GGsTop). Furthermore, we applied gGlu-HMRG to biopsy specimens which were taken from antrum and corpus of stomach in H. pylori positive patients $(\mathrm{n}=17)$ and H. pylori negative patients $(\mathrm{n}=19)$. We then observed the increase of fluorescence intensity over time (1 $\mathrm{min}, 5 \mathrm{~min}, 10 \mathrm{~min}, 15 \mathrm{~min}$ ). Fluorescence intensity was quantified by Image $\mathrm{J} 2$ software (National Institutes of Health, Rockville, Maryland).

Result: Activation of gGlu-HMRG fluorescence was detected in WT strain, but was not in ggt mutant strain. Activation of gGluHMRG fluorescence was inhibited by GGsTop. gGlu-HMRG fluorescence significantly increased $H$. pylori positive mucosa compared with $H$. pylori negative mucosa. There was significant difference of fluorescence in both specimens from antrum and corpus (antrum $\mathrm{p}=$ 0.000073 , corpus $=0.0089$ ).
Conclusion: GGT-activated fluorescent probe can be useful for H. pylori infection rapid diagnosis.

\section{3-2 \\ LncRNA THRIL is Involved in the Phosphorylation of CagA and Regulates Helicobacter pylori CagA Induced- Inflammation}

\author{
Na Keum Lee, Jung-Ho Youn, Kyu Yeon Hahn, Sang Kil Lee \\ Institute of Gastroenterology Department of Internal \\ Medicine, Yonsei University College of Medicine \\ Severance Hospital Seoul Korea
}

Introduction: The most obvious cause of gastritis and gastric cancer is known as Helicobacter pylori (H. pylori) infection and cytotoxin-associated gene A (CagA). Although there have been relatively many studies on how cagA actually causes disease, there is limited research into how the activity of cagA is regulated in humans. Recently, long non-coding RNA is interestingly emerged to be associated with regulation of the immune response by pathogen. THRIL (TNF $\alpha$ and hnRNPL related immunoregulatory lincRNA) was first reported to regulate lipopolysaccharide induced tumor necrosis factor alpha $(\mathrm{TNF} \alpha)$ by interacting with heterogenous nuclear ribonucleoprotein L (hnRNPL). We investigated the roles of THRIL in H. pylori cagA induced-infection.

Results: The level of THRIL expression was higher in stomach than other organs. H. pylori strain $60190(\mathrm{CagA}+)$ induced TNF $\alpha$ and IL-8 in GES-1 cell, and its expression is inversely down-regulated to THRIL. H. pylori strain 8822 (CagA-) and $\Delta$ CagA did not influence the levels of TNF $\alpha$, IL-8 and THRIL. Down regulation of THRIL decreased mRNA expression of TNF $\alpha$ and IL-8 in both basal status and CagA-positive H. pylori infection significantly, respectively. Also, double knockdown of THRIL and hnRNPL significantly attenuated TNF $\alpha$ induction by CagA-positive $H$. pylori. A series of cellular process is preceded in that THRIL and hnRNPL regulated CagA-induced inflammation by NF-kB translocation. In addition, Knockdown of THRIL reduced c-Abl, which plays an important role in cagA phosphorylation and activation, and thus also significantly decreased phosphorylation of cagA.

Conclusion: THRIL is expected to play an important role in the disease caused by Helicobacter. $\overline{106} \quad \overline{\text { Digestion 2019;99:95-119 }}$

DOI: $10.1159 / 000494404$
The 11th International Gastrointestinal Consensus Symposium (IGICS) 


\section{3-3 \\ Walnut Phenolic Extract Alleviates \\ Non-Steroidal Anti-Inflammatory \\ Drug-Induced Gastric Mucosal Damages \\ by Increasing Cyclooxygenase-2 Inhibition, 15-Hydroxyprostaglandin Dehydrogenase Induction, and Heme Oxygenase-1 Induction}

Jong-Min Park', Young-Min Han', Eun-A Kang ${ }^{1}$, You Kang ${ }^{1}$, In Kyung Cho, Seong Pyo Hong, Ki-Baik Hahm ${ }^{1,2}$

${ }^{1} \mathrm{CHA}$ Cancer Prevention Research Center, CHA Cancer

Institute, CHA University, Seoul, Korea; ${ }^{2}$ Digestive

Disease Center, CHA University Bundang Medical Center, Seongnam, Korea

Backgrounds: Non-steroidal anti-inflammatory drugs (NSAIDs), the most highly prescribed drugs in the world for the treatment of pain, inflammation, and fever, are associated with gastric mucosal damages including ulcer directly or indirectly. This study was aimed to document the preventive effects of walnut phenolic extract (WPE), against NSAIDs-induced gastric damages, as well the elucidation of its pharmacological actions, such as anti-inflammatory, anti-oxidative, and cytoprotective actions.

Methods: RGM-1 gastric mucosal cells were administered with NSAIDs and compared the expressions of inflammatory mediators after indomethacin alone or combination of indomethacin and WPE. The expressions of inflammatory mediators, including cyclooxygenase-2, cyclooxygenase-1, prostaglandin E2, 15-hydroxyprostaglandin dehydrogenase, and anti-oxidant capacity, were analyzed by Western blot analysis, RT-PCR, and ELISA, respectively. Detailed documentation of in vitro biological actions of WPE, including phase 2 enzymes, was also performed.

Results: NSAID increased the expression of COX-2 and decreased COX-1 and 15-PGDH, but WPE significantly attenuated NSAID-induced COX-2 expression. Interestingly, WPE induced expression of 15-PGDH. By using deletion constructs of the 15-PGDH promoter, we have found that c-Jun is the most essential determinant for WPE-induced up-regulation of 15-PGDH expression. We confirmed that knockdown of c-Jun abolished the ability of WPE to up-regulate 15-PGDH expression. In addition, WPE significantly increased HO-1 expression. WPE increased nuclear translocation of Nrf2 by Keap-1 degradation and silencing Nrf2 markedly reduced the WPE-induced HO-1 expression. We have found that WPE-induced HO-1 up-regulation was attenuated in cells harbouring the mutant Keap1 in which the cysteine 151 residue was replaced by serine.

Conclusions: WPE can be promising therapeutics agent to provide potent anti-inflammatory, anti-oxidative, and mucosa protective effects against NSAID-induced damages.

\section{3-4 \\ Placenta Derived Mesenchymal Stem Cells Afforded Rejuvenation of Helicobacter pylori-Associated Atrophic Gastritis Better Than Cord Derived or Adipose Tissue Derived MSCs}

\author{
Jong Min Park, Young Min Han, Shin A Shin, \\ Seong Pyo Hong, Eun A Kang, Weon Jin Ko, \\ Joo Young Cho, Ki Baik Hahm \\ CHA Cancer Prevention Research center, CHA University, \\ Pangyo, Korea; Digestive Disease Center, CHA University \\ Bundang Medical Center, Korea
}

Supported with significant rejuvenating and regeneration actions of human placenta derived mesenchymal stem cells (hPDSCs), we have explored detailed underlying molecular mechanisms specific to stem cells. In this study that $H$. pylori-initiated, high salt diet-promoted gastric carcinogenesis model, we have administered hPDSCs around 15-20 weeks after $H$. pylori infection and sacrificed mice at 24 and 48 weeks, respectively in order to either assess the pathological conditions or explore typical molecular changes. At 24 weeks, significant changes were attenuated 8 weeks, significant attenuations were appearance of gastric dysplasia and tumors. In detail, gastric inflammatory mediators, apoptosis, and mucosal change biomarkers were significantly changes with hPDSCs, especially Raman spectroscopy showed significant changes in stem cell treated group, SERS findings. 15-PGDH, as well-acknowledged tumor suppressive enzyme, were significantly preserved with hPDSCs, whereas significantly decreased in $H$. pylori control group $(\mathrm{p}<0.01)$. Stanniocalcin-1 (STC-1) and thrombospondin-1 (TSP-1) were known as good biomarkers reflecting stem cell activities. Compared to control group where STC-1 and TSP-1 were significantly decreased, their expressions were significantly increased in hPDSC treated group. Though similar MSCs, umbilical cord derived mesenchymal stem cell (hCD$\mathrm{SCs}$ ) and adipose tissue derived MSCs (hSDSCs), the biological actions of rejuvenating $H$. pylori-associated atrophic gastritis were only significantly noted in hPDSCs along with significant expressions of STC-1 and TSP-1. Inflammasome and efferocytosis were significantly regulated with hPDSCs. In conclusion, hPDSCs can be of potential agent to rejuvenate $H$. pylori-associated atrophic gastritis and STC-1 and TSP-1 can be good biomarkers to predict response. 


\section{3-5 \\ Microbial Compositional and Ecological Dysbiosis in Gastric Carcinogenesis}

Olabisi Oluwabukola Coker ${ }^{1}$, Zhenwei Dai ${ }^{*}$, Geicho Nakatsu', Sunny Hei Wong ${ }^{1}$, William Ka Kai Wu', Yongzhan Nie", Guijun Zhao ${ }^{3}$, Lei Cao', Zigui Chen ${ }^{4}$, Joseph JY Sung ${ }^{1}$, Jun Yu ${ }^{1}$

${ }^{1}$ Institute of Digestive Disease and Department of Medicine and Therapeutics, State Key Laboratory of Digestive Disease, Li Ka Shing Institute of Health Sciences, CUHK Shenzhen Research Institute, The Chinese University of Hong Kong, Hong Kong; ${ }^{2}$ State Key Laboratory of Cancer Biology, Xijing Hospital, Fourth Military Medical University, Xi'an, China; ${ }^{3}$ Department of Gastroenterology and Hepatology, Inner Mongolia People's Hospital, Hohhot, China; ${ }^{4}$ Department of Microbiology, Faculty of Medicine, The Chinese University of Hong Kong, Hong Kong

Introduction: Gut microbiota dysbiosis is associated with gastrointestinal diseases. We aimed to characterize microbial compositional and ecological changes associated with progressive histological stages of gastric tumorigenesis.

Methods: We performed 16S rRNA gene analysis of gastric mucosal samples from 81 cases including superficial gastritis (SG), atrophic gastritis (AG), intestinal metaplasia (IM) and gastric cancer (GC) from Xi'an, China, to determine mucosal microbiome dysbiosis across stages of GC. We validated the results in mucosal samples of 126 cases from Inner Mongolia, China.

Result: We observed significant mucosa microbial dysbiosis in IM and GC subjects, with significant enrichment of 21 and depletion of 10 bacterial taxa in GC compared to SG $(q<0.05)$. Microbial network analysis showed increasing correlation strengths among them with disease progression $(\mathrm{p}<0.001)$. Five $\mathrm{GC}$-enriched bacterial taxa whose species identifications correspond to Peptostreptococcus stomatis, Streptococcus anginosus, Parvimonas micra, Slackia exigua and Dialister pneumosintes had significant centralities in the GC ecological network $(p<0.05)$ and classified GC from SG with an area under the receiver-operating curve (AUC) of 0.82. Moreover, stronger interactions among gastric microbes were observed in Helicobacter pylori-negative samples compared to $H$. pylori-positive samples in SG and IM. Metagenomics functional prediction showed that nucleotide metabolism, peptidoglycan biosynthesis and carbohydrate digestion and absorption were significantly higher in GC microbiota, while proteins associated with host bacterial recognition were depleted. The GC-associated microbial functional changes, fold changes of selected bacteria and strengths of their interactions were successfully validated in the Inner Mongolian cohort, in which the five bacterial markers distinguished GC from SG with an AUC of 0.81 .

Conclusion: In addition to microbial compositional and functional changes, we identified differences in bacterial interactions across stages of gastric carcinogenesis. The significant enrichments and network centralities suggest the potentially important roles of $\mathrm{P}$. stomatis, D. pneumosintes, S. exigua, P. micra and S. anginosus in GC progression.

\section{3-6 \\ Extensive Gastric Mucosal Atrophy May Prevent from Superficial Non-Ampullary Duodenal Epithelial Tumor (SNADET)}

\author{
Yohei Koyama1, Takashi Kawai², Kumiko Uchida', \\ Akira Madarame1, Takayuki Morise, Yuki Aoki', \\ Akihiko Sugimoto ${ }^{1}$, Yuichiro Tsuji ${ }^{1}$, Kenji Yagi ${ }^{1}$, Takao Itoi ${ }^{1}$ \\ ${ }^{1}$ Department of Gastroenterology and Hepatology، \\ ${ }^{2}$ Department of Endoscopy center, Tokyo Medical \\ University, Japan
}

Background: Gastric mucosal atrophy due to Helicobacter pylori infection is well known as a risk factor for gastric cancer. However, the risk factor for SNADET has been obscure.

Aims: To clarify whether gastric mucosal atrophy is associated with SNADET.

Methods: We conducted a retrospective study on consecutive 59 SNADET patients (41 male/18 female: Group A) who underwent endoscopic resection between January 2009 and February 2017 at our institution. On the other hand, a control matched by sex and age group (Group B) was randomly selected to compare with Group A from among patients who underwent endoscopies as screening during the same period. The macroscopic extent of gastric mucosal atrophy was compared between 2 groups. The macroscopic extent of gastric mucosal atrophy was classified into 6 groups by Kimura-Takemoto classification (non-atrophic or $\mathrm{C} 1, \mathrm{C} 2, \mathrm{C} 3, \mathrm{O} 1, \mathrm{O} 2$, and $\mathrm{O} 3 ; \mathrm{C}$, closed; O, open) each with, 0 to 5 points respectively.

Results: The mean age of Group A was $60.5 \pm 10.5$ years. The macroscopic extent of gastric mucosal atrophy (non-atrophic or $\mathrm{C} 1$, C2, C3, O1, O2, and O3) were in Group A: 41, 3, 4, 5, 6, and 0 cases, and in Group B:29, 3, 6, 8, 8, and 5 cases, respectively. The mean score of gastric mucosal atrophy was $0.85 \pm 1.41$ points in Group A, and $1.63 \pm 1.82$ points in Group B, respectively. Group A had significantly mild gastric mucosal atrophy compared to Group B $(\mathrm{p}=0.01)$.

Conclusions: Score of gastric mucosal atrophy was lower in SNADET than that of control group. We speculate that extensive gastric mucosal atrophy causes to reduce gastric acid secretion and it may decreases SNADET incidence. Further investigations including the presence of $H$. pylori are needed in the near future.
The 11th International Gastrointestinal Consensus Symposium (IGICS) 


\section{Oral Session 4}

\author{
Chairpersons: Tetsuya Mine (Tokai University, Japan) \\ Jose D. Sollano (University of Santo Tomas, \\ Manilia, Philippines)
}

\section{4-1 \\ P-CAB (Revaprazan) Prevented Indomethacin-Induced Permeability Through Tightening TJs}

\author{
Young-Min Han ${ }^{1}$, Jong-Min Park', Joo Yong Cho ${ }^{2}$, \\ Yoo Kang ${ }^{1}$, Seong Pyo Hong ${ }^{2}$, Deok Hwan Kim², \\ Ki-Baik Hahm ${ }^{1,2}$ \\ ${ }^{1} \mathrm{CHA}$ Cancer Prevention Research Center, CHA Cancer \\ Institute, CHA University, Seoul, Korea; ${ }^{2}$ Digestive \\ Disease Center, CHA University Bundang Medical center, \\ Seongnam, Korea
}

Non-steroidal anti-inflammatory drugs (NSAIDs) are the most commonly prescribed drugs for arthritis, inflammation, and cardiovascular protection. However, they cause gastrointestinal side effects. The pathophysiology of these complications has mostly been originated on the cyclooxygenase inhibition and the subsequent prostaglandin deficiency as action of NSAIDs. However, recent clinical data showed that PPI aggravated NSAIDs-induced intestinal damages through either dysbiosis or increased intestinal permeability, suggesting PPI might be no more protective against NSAID-induced intestinal damages. In this study, we investigated the changes of indomethacin (IND)-induced small intestinal damages after combination with pantoprazole (PPZ) or combination with revaprazan, potassiumcompetitive acid blocker (REV, P-CAB). All SD rats were 5 weeks of age. After treatment after 48 hours rats with indomethacin in all the groups, they were euthanized and small intestine were collected for gross and pathologic evaluation, followed by immunohistochemical staining, Western blotting for inflammatory mediators, permeability protein, and RT-PCR. As a result, macroscopic and histological evidences suggested that administration of IND resulted in significant intestinal damage and co-administration of PPZ resulted in worsen of IND enteropathy while attenuation of IND-induced intestinal damage in co-administration of REV. Moreover when we checked inflammatory cytokines and oxidative stress-associated factors using Western blotting and RT-PCR showed that IND with PPZ group was higher compared to IND alone group but IND plus REV group was lower compared to the PPZ group. Also we performed immunohistochemical staining to confirm intestinal permeability including ZO-1 and Occludin 1 as a result their expression was significantly decreased in IND with PPZ group, whereas no changes in IND + REV group, suggesting that PPZ aggravated IND-induced small intestinal damages, but not with REV combination. Thus this study in the near future that P-CAB should be selected to secure NSAID-induced GI damages, while PPI should not be prescribed in this purpose.

\section{4-2 \\ Verification of Intestinal Microflora- Mediated Mucosal Homeostasis by Immunoglobulin A}

\author{
Takashi Nagaishi", Taro Watabe ${ }^{1}$, Akinori Hosoya ${ }^{1}$ \\ Jose Nisha', Yudai Kojima', Takahiro Adachi², \\ Mamoru Watanabe ${ }^{1}$
}

${ }^{1}$ Department of Gastroenterology, Graduate School of Medical Science, Tokyo Medical and Dental University, Japan; ${ }^{2}$ Department of Immunology, Medical Research Institute, Tokyo Medical and Dental University, Japan

Background and Aim: Immunoglobulin (Ig) A is believed to be involved in the maintenance of mucosal homeostasis. In fact, IgA deficiency has been associated with gastrointestinal diseases such as celiac disease and inflammatory bowel diseases. In addition, it has been reported that the lack of Ig class-switching in B cells due to the deficient expression of activation-induced cytidine deaminase in mice $\left(\mathrm{AID}^{-/-}\right.$) results in aberrant microflora composition in the gut. However, it is still unclear whether this is caused by the lack of IgA since $\mathrm{AID}^{-/}$also results in deficiency of other Ig classes. Here, we verified the $\operatorname{IgA}$ regulation of intestinal microflora.

Methods and Results: We have recently generated an animal model of IgA defficiency (IgA ${ }^{-/}$) by using the CRISPR/Cas9 system with the guide RNAs specific for IgE and IgA cytoplasmic domains, and subsequently confirmed substantial expressions of all Ig classes except IgA. Scanning electron microscopy showed significantly increased segmented filamentous bacteria on the intestinal epithelia in $\mathrm{IgA}^{-/-}$compared to littermate control. Metagenomic analysis using microbial 16S rRNAs isolated from the luminal contents of jejunum, ileum, colon or fecal samples revealed significantly altered microflora diversity especially in the ileum. Moreover, histopathology and flow cytometry revealed inflamed mucosa and increased lamina propria $\mathrm{CD}^{+} \mathrm{T}$ cells in the ileum, respectively, and no significant changes at the other alimentary tracts. These were associated with increased IFN-g and IL-17 and decreased IL-4 production. $\operatorname{Ig} \mathrm{A}^{-/-}$were then intercrossed with CD19Cre-YC3.60 transgenic mice to analyze $\mathrm{Ca}^{2+}$ influx. Intra-vital imaging interestingly revealed activated B cells in Peyer's patches at the ileum.

Conclusion: Our study provides first evidence to verify that the lack of IgA induces altered microflora as previously observed in $\mathrm{AID}^{-/}$. In addition, our observations also imply that such alteration may result in mucosal inflammation specifically in the ileum caused by dysregulation of homeostasis. 


\section{4-3 \\ Pathogenic Role of Dysbiosis in the Development of 5-Fluorouracil-Induced Intestinal Mucositis in Mice}

Nahla Hamouda', Tetsushi Sano², Masaki Shimakawa³, Kenjiro Matsumoto', Kikuko Amagase', Kazuhide Higuchi², Shinichi Kato

'Division of Pathological Sciences, Department of Pharmacology and Experimental Therapeutics, Kyoto Pharamceutical University, Kyoto, Japan; ${ }^{2}$ Second Department of Internal Medicine, Osaka Medical College, Osaka, Japan; ${ }^{3 B}$ iofermin Pharmaceutical Co., Ltd. Hyogo Japan

Introduction: The chemotherapeutic agent 5-fuorouracil (5-FU) causes intestinal mucositis with severe diarrhea but the pathogenesis is not fully understood. In the present study, we investigated the pathogenic effects of 5-FU in mice, focusing on enterobacteria, apoptosis and inflammatory cytokines.

Methods: Male C57BL/6 mice were given $50 \mathrm{mg} / \mathrm{kg} 5-\mathrm{FU}$ by intraperitoneal injection once daily for six days. Ampicillin $(250 \mathrm{mg} /$ $\mathrm{kg})$ and aztreonam $(50 \mathrm{mg} / \mathrm{kg})$ were co-administered orally twice daily for six days. Disease severity was assessed daily by measuring bodyweight and scoring stool consistency (0-4). On day 1, 2, 4 and 6 following exposure to 5-FU, the jejunum was collected, tissue samples were excised and examined for MPO activity, inflammatory cytokines and stained for histology and apoptosis. Fecal samples were collected and analysed for DNA microbiota.

Result: Repeated administration of 5-FU caused severe intestinal mucositis, characterized by shortening of villi and destruction of crypts. TUNEL-positive apoptotic cells were detected in crypts on day 1 but this response was diminished from day 2 and thereafter. The evident increase in MPO activity and IL-1 $\beta$ expression was observed from day 4 while upregulation of TNF- $\alpha$ expression was detected on day 1 and further augmented from day 4. Daily administration of ampicillin, a broad-spectrum antibiotic, and aztreonam, a gram-negative bacteria-selective antibiotic, significantly reduced the severity of intestinal mucositis, the increase in MPO activity, and cytokine upregulation on day 6 without any effect on apoptosis induction and upregulation of TNF- $\alpha$ on day 1 following 5-FU treatment. The analysis of intestinal microbiota showed that 5-FU treatment caused disruption of the microbiota, notably decreasing Firmicutes and increasing Bacteroides, but these changes were improved by daily administration of ampicillin.

Conclusion: These findings suggest that dysbiosis accompanied by crypt apoptosis and inflammatory cytokines are involved in the occurrence of 5-FU-induced intestinal mucositis. The apoptosis induces dysbiosis via disruption of epithelial barrier, resulting in upregulation of inflammatory cytokines.

\section{4-4 \\ Endoscopic Allpication of New Hemostatic Powder (UI-EWD ${ }^{\circledR}$ ) in Gastrointestinal Bleeding}

\author{
Yong Woon Shin, Byung Wook Bang
}

Department of Gastroenterology Inha University School of Medicine, Incheon, Korea

Introduction: Although endoscopic hemostasis is usually effective in controlling gastrointestinal (GI) hemorrhage, some have difficulty in achieving successful hemostasis depending on the location and severity of hemorrhage. UI-EWD ${ }^{\circledR}$ (Next Biomedical, ncheon, South Korea is a highly adhesive powder and the hemostatic effects are accomplished when the UI-EWD ${ }^{\circledR}$ powder immediately forms a hydrogel when contacting the water. It shows high adhesiveness and persistency on bleeding site. In addition, we developed new spray device which reduce catheter clogging during spray.

Aims and Methods: This was a prospective study investigating the efficacy of UI-EWD in patients with various gastrointestinal bleeding. The UI-EWD powder was applied to post-ESD/EMR ulcer or to the bleeding sites undergoing insufficient hemostasis. We evaluate 1) the success rate of hemostasis 2) re-bleeding rate on secondlook endoscopy at the next day, 3) Persistent rate of UI-EWD on ulcer base at follow-up endoscopy, and 4 clogging rate of spraying catheter during applying UI-EWD ${ }^{\circledR}$.

Results: A total of 57 patients were enrolled. The bleeding developed in 46 patients with post-endoscopic resection ulcers (41 ESD induced ulcers and 5 EMR induced ulcers), 8 patients with peptic ulcers and 3 patients with miscellaneous bleeding. 1) Success rates of hemostasis in acute bleeding were $94.8 \%(55 / 58)$ of UI-EWD ${ }^{\circledR}$ group. 2) Re-bleeding rates were $6.9 \%(4 / 58)$ of UI-EWD ${ }^{\circledR}$ group. Persistent rate of UI-EWD ${ }^{\circledR}$ on ulcer base was $70.2 \%(26 / 37) 1$ day after the procedure, and $38.4 \%$ (15/38) 3 days after the procedure. 4$)$ Clogging rate of spraying catheter was $4.4 \%(2 / 45)$.

Conclusions: The endoscopic application of UI-EWD ${ }^{\circledR}$ is effective for the acute GI bleeding. The hemostatic action of UI-EWD appears to be from high mucoadhesiveness and hydrogel persistence. In addition, a newly developed delivery system shows less scattering and more targeted properties onto ulcer base.
The 11th International Gastrointestinal Consensus Symposium (IGICS) 


\section{4-5 \\ Health-Related Quality of Life and Personal Living Status among Patients with Inflammatory Bowel Disease: A Multicenter Investigation in Mainland China}

\begin{abstract}
Chenwen Cai', Zhihua Ran ${ }^{1}$, Meilan Huang ${ }^{1}$, Naizhong Hü, Yihong Fan ${ }^{3}$, Chengdang Wang ${ }^{4}$, Chunxiao Chen ${ }^{5}$,

Qing Zheng', Tianrong Wang', Yuqi Qiao',

Mingming Zhu', Zhanghan Dai', Ying Sun', Di Zhao', Dongjuan Song', Lijie Lai', Mi Zhou', Jun Shen ${ }^{1}$

${ }^{1}$ Division of Gastroenterology and Hepatology,Key Laboratory of Gastroenterology and Hepatology, Ministry of Health, Inflammatory Bowel Disease Research Center,Renji Hospital, School of Medicine, Shanghai Jiao Tong University; Shanghai Institute of Digestive Disease; China; ${ }^{2}$ Department of Gastroenterology, The First Affiliated Hospital of Anhui Medical University, Key Laboratory of Gastroenterology of Anhui Province, China; ${ }^{3}$ Department of Gastroenterology, The First Affiliated Hospital of Zhejiang Chinese Medical University, China; ${ }^{4}$ Department of Gastroenterology, The First Affiliated Hospital of Fujian Medical University, China; ${ }^{5}$ Department of Gastroenterology, The First Affiliated Hospital, Medical College of Zhejiang University, China
\end{abstract}

Background and Aims: To investigate health-related quality of life (HRQOL) and personal living status of patients with inflammatory bowel disease (IBD) in multiple clinical centers in mainland China.

Methods: Short inflammatory bowel disease questionnaire (SIBDQ) and patient-reported 0 to 10 score of IBD (IBD-10) were applied to evaluate HRQOL meanwhile patients' family members who devoted most in caring were also asked to give an IBD-10 score to reflect their viewpoints towards patients' conditions. Patients' personal living statusincluding education, employment, income, marriage and childbearingwas surveyed.

Results: SIBDQ scores were lower in UC $(50,40-59)$ than in CD $(53,44-61)(p=0.009)$ while IBD-10 remained similar between two groups $(p=0.117)$. The proportion of post-secondary school education (college, university and graduate school) was higher in IBD participants cohort (51.4\%) than in general population $(28.1 \%)(\mathrm{p}<$ $0.0001)$ however unemployment rate of patients $(31.8 \%)$ was also much above the population rate $(p<0.0001)$. The marriage rate was higher in female than in male patients $(p=0.013)$ while the divorce rate had no significant difference $(p=0.228)$. The total marriage and divorce rates of IBD patients were quite similar to the rates of city population ( $p=0.357$ ) however female individuals got a higher marriage rate than the average $(p=0.043)$.

Conclusion: Patients' family members who are most responsible for caring may form a similar perception towards disease as patients do. Compared with general population data, IBD patients appear to achieve higher educational levels but also obtain a higher unemployment rate, which should arouse the attention of the entire society.

\section{4-6 \\ A Case of Esophageal Motility Disorder in Systemic Sclerosis Treated by Acotiamide}

Tsutomu Takeda', Kenshi Matsumoto ${ }^{2}$, Akihito Nagahara', Yoichi Akazawa², Hiroyuki Komori², Kentaro Izumi², Kohei Matsumoto", Hiroya Ueyama², Yuji Shimada', Daisuke Asaoka', Mariko Hojo², Sumio Watanabe²

1 Juntendo University Juntendo Tokyo Koto Geriatric Medical Center, Japan; 'Juntendo University school of

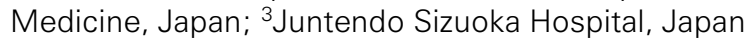

Case: A woman in her sixties with systemic sclerosis (SSc) was referred as an outpatient for assessment of dysphasia and reflux symptom. She had been treated with $9 \mathrm{mg}$ of oral PSL and proton pump inhibitors, but PPIs did not take effect. The Eckardt score was six and the Dysphagia score was two. A high-resolution manometry (HRM) found loss of Segment 2 or 3 peristaltic waves. She was diagnosed with absent contractility and DCI (mm Hg-cm-s) was low (101.7). Another HRM after one month of treatment with acotiamide (300 $\mathrm{mg} /$ day) found $\mathrm{DCI}=478.3$ and an increase of $\%$ peristaltic from zero to 63 , and the symptoms scores improved, with Eckardt score/ Dysphagia score $2 / 0$. In order to compare the effects with mosapride, after withholding acotiamide for one month for washout, the medication was switched to mosapride (15 mg/day). The results of HRM one month later were similar to those of acotiamide (DCI $=453.7)$. However, the symptom scores changed for the worse, with $4 / 2$, respectively. Acotiamide was restarted after withholding mosapride for one month to confirm reproducibility. The improvements of the symptom scores $(2 / 1)$ were observed with DCI $=477.1$.

Discussion/Conclusion: Both acotiamide and mosapride improved \% peristaltic, and acotiamide notably improved the symptoms scores. From previous studies, both acotiamide and mosapride are known to improve gastric adaptive relaxation and gastric emptying. However, they are believed to be different in that acotiamide directly increases Ach by its AChE inhibitory action and improves gastrointestinal tract motility. On the other hand, mosapride indirectly increases the production of Ach by its serotonin 5-HT4 receptor agonistic action. These differences may have caused the difference in the effects of these drugs. We have experienced a case suggesting that acotiamide may be effective treatment to improve the esophageal motility and the symptoms in SSc. 


\section{IGICS Poster Session 1}

\author{
Chairperson: Qi Zhu (Shanghai Jiao Tong University \\ School of Medicine, China)
}

\section{IP1-1 \\ Clinical Efficacy \& Safety of Mosapride in Functional Dyspepsia Patients}

\author{
Dadang Makmun, Murdani Abdullah, Hasan Maulahela, \\ Saskia Aziza, A Aziz Rani
}

Division of Gastroenterology, Department of Internal Medicine, Faculty of Medicine, University of Indonesia, Dr Cipto Mangunkusumo, Indonesia

Introduction: Functional dyspepsia is a common disorder in gastroenterology clinical practice, the pathophysiology of this condition is poorly understood and optimal therapeutic approaches are yet to be determined. Prokinetic agents have shown variable efficacy in the treatment of functional dyspepsia. Mosapride is a novel 5-hydroxytryptamine-4 (5-HT4) agonistic compound, and its primary metabolite, M1, has 5-HT4 agonistic as well as 5-HT3 antagonistic properties. Aim of this study was to determine the efficacy and safety of mosapride in functional dyspepsia.

Methods: This is a single open label, pre and post treatment in a single-centre study. All dyspeptic patients who underwent to gastroenterology polyclinic in dr. Cipto Mangunkusumo Hospital Hospital were being studied. The dyspeptic patients are followed on first day and day 28 with Visual Analogue Scale (VAS). The subjects administered mosapride with safety laboratory examination on first day and follow up in day 28th.

Results: Sixty six subjects enrolled (55 complete and 12 drop out subject). From 55 subjects showed female (74.5\%; 41 subjects) is more frequent than male $(25.5 \% ; 14$ subject). Scores of dyspepsia showed different value before and after treatment of intervention, pre and post treatment of nausea ( 4.15 vs $1.05 ; \mathrm{p}<0.05)$, vomiting $(1.27$ vs $0.04 ; \mathrm{p}<0.05$ ), anorexia ( 1.56 vs $0.31 ; \mathrm{p}<0.05)$, bloating ( 4.87 vs $1.24 ; \mathrm{p}<0.05)$, belching (4.16 vs $1.18 ; \mathrm{p}<0.05)$, early satiety $(2.58$ vs $0.62 ; \mathrm{p}<0.05)$. Laboratory examination pre and post treatment showed no statistically significant change except bilirubin ( 0.66 vs $0.58 ; \mathrm{p}<0.05)$ and neutrophil $(65.15$ vs $61.80 ; \mathrm{p}<0.05)$ but in clinically not significant. No adverse event reported in this study.

Conclusion: Mosapride as a prokinetic agent has a significant role in treating functional dyspepsia symptoms and good safety profile.

\section{IP1-2 \\ New Paradigm for Cancer Prevention; Dietary Kimchi Prevents Inflammation and Cancer by Helicobacter pylori in vitro}

Yoo Kang ${ }^{1}$, Young-Min Han' ${ }^{1}$ Ji-Young Oh², Jong-Min Park', Dong-Yoon Lee ${ }^{2}$, In Kyung Yoo ${ }^{3}$, Seong Pyo Hong ${ }^{3}$, Ki-Baik Hahm ${ }^{1,3}$

${ }^{1} \mathrm{CHA}$ Cancer Prevention Research Center, CHA Cancer Institute, CHA University, Seoul, Korea; ${ }^{2}$ CJ Food, CJ Blossom Park, Suwon, Korea Digestive Disease Center, CHA University Bundang Medical Center, Seongnam, Korea

Purpose: To prove whether dietary intervention can prevent Helicobacter pylori (H. pylori - induced atrophic gastritis and gastric cancer, we developed cancer preventive kimchi (cpKimchi) through special recipe and treated to $H$. pylori-infected cells or gastric cancer cells. We have compared anti-inflammaotry, anti-oxidative, and cytoprotective actions of cancer preventive kimchi against H.pylori infection in vitro.

Methods: The paper disc assay to check anti-microbial action and RT-PCR and Western blot were performed to check anti-inflammatory action. Cancer preventive kimchi treated 1 hour before $H$. pylori infection on AGS cells. And we also checked effects for cancer inhibition after cpKimchi-treatment in MKN28 gastric cancer cells.

Results: The cpKimchi had fabulous efficacy on antimicrobial action which was further confirmed by paper disc assay with H.pylori. The expressions of COX-2, iNOS, IL6 and VEGF were increased after $H$. pylori infection, of which levels were significantly decreased with cpKimchi. And cpKimchi significantly increased HO1, NQO1, SOD and GST (pi) expression as anti-oxidative enzymes. Moreover MTT assay was done in MKN28 cells under the challenge with 20 $\mathrm{mg} / \mathrm{ml}$ concentration of cpKimchi. Significant cyototoxicities were noted with cpKimchi in MKN28 cells. This decrease in cell viability was confirmed by induction of apoptosis by cpKimchi treatment using Westernblotting.

Conclusion: The cpKimchi can be anticipating substance afforded significant attenuation of either $H$. pylori-induced gastritis or tumorigenesis based on potent anti-oxidative, anti-inflammatory, and anti-mutagenic actions. 


\section{IP1-3 \\ Walnut Phenolic Extract Inhibited Helicobacter pylori-Induced STAT3 Phosphorylation Through Activation of PPAsRy}

Eun-A Kang ${ }^{1}$, Jong-Min Park', Young-Min Han', You Kang ${ }^{1}$, In Kyung Yoo ${ }^{1}$, Seong Pyo Hong ${ }^{1}$, Joo Young Cho', Ki-Baik Hahm ${ }^{1,2}$

${ }^{1} \mathrm{CHA}$ Cancer Prevention Research Center, CHA Cancer Institute, CHA University, Seoul, Korea; ${ }^{2}$ Digestive Disease Center, CHA University Bundang Medical Center, Seongnam, Korea

Backgrounds: The health beneficial effects of walnut phenolic extract (WPE) have been attributed to its anti-inflammatory properties. However, the molecular mechanism underlying anti-inflammatory effects of WPE remains largely elusive.

Methods: Recent studies have shown that Helicobacter pylori (H. pylori) activates signal transducer and activator of transcription 3 (STAT3) that plays an important role in gastric carcinogenesis. In the present study, AGS cells were cocultured with $H$. pylori, and STAT3 activation was assessed by Western blot analysis and immunocytochemistry. WPE was found to suppress the phosphorylation and nuclear translocation of signal transducer and activator of transcription 3 (STAT3) induced by Helicobacter pylori infection in human gastric cancer AGS cells.

Results: Notably, WPE induced expression of suppressor of cytokine signaling 1 (SOCS1), a negative regulator of STAT3. Knockdown of SOCS1 abolished the suppressive effect of WPE on STAT3 (Tyr705) phosphorylation induced by $H$. pylori infection. WPE also induced nuclear translocation and transcriptional activities of peroxisome proliferator-activated receptor gamma (PPAR $\gamma$ ) in AGS cells. Knockdown of PPAR $\gamma$ inhibited the transcription of SOCS1 and attenuated the suppressive effect of WPE on phosphorylation of STAT3 (Tyr705) induced by H. pylori. The PPAR $\gamma$ antagonist bisphenol A diglycidyl ether also mitigated the suppressive effect of WPE on H. pylori-induced phosphorylation of STAT3 (Tyr705). In addition, WPE inhibited the expression of c-Myc, which was attenuated in the AGS cells harboring SOCS1 specific siRNA. WPE also markedly decreased anchorage-independent growth of AGS cells infected by $H$. pylori.

Conclusions: WPE inhibits $H$. pylori-induced STAT3 phosphorylation in a PPAR $\gamma /$ SOCS1-dependent manner.

\section{IP1-4 \\ Modified Docetaxel + Cisplatin + Capecitabine (DCX) for Gastric Cancer}

Osamu Maeda1,2, Yuichi Ando', Ayumu Matsuoka1,2, Kazuhiro Furukawa², Kohei Funasaka², Ryoji Miyahara², Yoshiki Hirooka², Hidemi Goto ${ }^{2}$

${ }^{1}$ Department of Clinical Oncology and Chemotherapy, Nagoya University Hospital, Japan; ${ }^{2}$ Department of Gastroenterology and Hepatology Nagoya University Graduate School of Medicine, Japan

Standardized treatment for Stage IV gastric cancer is combination of platinum agent and fluoropyrimidine. To enforce effectiveness of the treatment, various triplet regimens adding taxanes have been tried. On the other hand, such triplet treatments increase risk of adverse events including myelosuppression. We performed a feasibility study of modified DCX for Stage IV gastric cancer using docetaxel $30 \mathrm{mg}$ / $\mathrm{m}^{2}$ or $40 \mathrm{mg} / \mathrm{m}^{2}$ day 1 , cisplatin $60 \mathrm{mg} / \mathrm{m}^{2}$ day 1 , and capecitabine $2000 \mathrm{mg} / \mathrm{m}^{2} /$ day days $1-14$ every 3 weeks (World J Gastroenterol. 23 (6):1090-1097, 2017), which includes smaller amount of docetaxel compared with previous reports using $60-75 \mathrm{mg} / \mathrm{m}^{2}$ of docetaxel. No patients experienced severe adverse events including febrile neutropenia. Three out of eight patients who received DCX underwent conversion surgery and are alive without recurrence for over three years. Based on the results of this trial, we planned a Phase II trial for unresectable or recurrent disease, and a feasibility trial as preoperative treatment for disease of macroscopic type 4 and large type 3 or with multiple lymph node metastases. We will present the results of these ongoing studies and future perspectives.

\section{IP1-5 \\ Small Intestinal Lymphoma with Obstructive Ileus Requiring Surgery}

Saskia Aziza Nursyirwan, Ari Fahrial Syam

Division of Gastroenterology, Department of Internal

Medicine, Faculty of Medicine Universitas Indonesia/Cipto

Mangunkusumo Hospital, Indonesia

Introduction: Perforation, bleeding, and ileus are known complications of small intestinal lymphoma. The most common primary site of small intestinal lymphoma is the ileum, followed by the jejunum, and duodenum; which diffuse large B-cell lymphoma is the major histopathology of the findings.

Case Illustration: A 69 years old male presented with abdominal pain and vomiting for several days duration with acute intestinal obstruction. He was diagnosed with diabetes mellitus type 2 and chronic kidney disease stage III. On abdominal examination, distended abdomen and increase bowel sound were found, but there was no sign of palpable mass. On plain abdominal X ray, there was obstructive ileus on the level of small intestine. CT scan of abdomen showed invagination of distal ileum with a suspected mass in the ileum. There are also slight hepatomegaly and bilateral pleural effusion. On laparotomy, there was a mesenteric mass involving the terminal ileum. Right hemicolectomy was done. Histopathology 
revealed small intestinal non Hodgkin's lymphoma-diffuse large B cell lymphoma. Patient then treated with chemotherapy.

Conclusion: Lymphoma should be considered as the cause of small intestinal obstruction so proper management can be proceed.

IP1-6

\section{A Rare Cause of Obscure Occult} Gastrointestinal Bleeding by Giardia Lamblia

Pitulak Aswakull, Pairoj Janyangdikul', Varayu Prachayakuß ${ }^{3}$, Udom Kachintorn ${ }^{3}$

${ }^{1}$ Liver and Digestive Institute, Samitivej Sukhumvit Hospital, Bangkok, Thailand; '2Department of pathology, Samitivej Srinagarind hospital, Bangkok, Thailand; ${ }^{3}$ Siriraj GI Endoscopy Center, Division of Gastroenterology, Department of Internal Medicine, Faculty of Medicine, Siriraj Hospital, Mahidol University, Bangkok, Thailand

Introduction: Obscured gastrointestinal bleeding is one of the common encountered clinical presentations for gastroenterologists. The steps of investigations to obtain the diagnosis of bleeding etiology are slightly different among each health institutes. Video capsule endoscopy had higher sensitivity for diagnosis of small intestinal bleeding etiology with lower specificity when comparing to other modalities. This case report demonstrated a rare cause of obscure occult gastrointestinal bleeding.

Methods: Case report.

Result: A 56 year old female presented with progressive anemia with anemic symptoms in few months. She did not reported any overt gastrointestinal bleeding. She developed abdominal discomfort and bloating without any diarrhea for couple months. Her physical examination revealed pale conjunctivae, no jaundice with mild abdominal distension without any tenderness. Otherwise were normal. Her blood works showed white blood cell 8,952 with neutrophile $68 \%$, lymphocyte $24.5 \%$, monocyte $2.1 \%$, eosinophile $5.4 \%$ and her hemoglobin level was $9.0 \mathrm{~g} / \mathrm{dL}$ (reference range; $12.2-14.8 \mathrm{~g} / \mathrm{dL}$ ). The ferritin level was 8.0 (reference range; $13-150 \mathrm{ng} / \mathrm{mL}$ ) with serum iron (reference range; $37-145 \mathrm{mg} / \mathrm{dL}$ ). Her stool examinations showed no parasite with positive stool occult blood for three times. She underwent esophagogastroduodenoscopy and colonoscopy which showed negative findings. After that, she underwent video capsule endoscopy which revealed markedly edematous mucosa with diffusely blunting and whitish villi at jejunum with some small area of denuded mucosa (Figure1). Finally, she underwent single balloon assisted enteroscopy which the findings showed markedly edematous mucosa of jejunum with whitish spots (Figure 2 ). Multiple biopsies were taken from jejunum. The histopathology showed mild infiltration of lymphoplasma cells and scant eosinophil in edematous lamina propia, abundant Giardia Lamblia trophozoites are presented in the inter-villous space (Figure 3A,B). Thus, the diagnosis of severe Giardiasis was made. The patient was prescribed $2 \mathrm{gm}$. single dose of tinidazole altogether with $800 \mathrm{mg}$ albendazole per day for totally 14 days, altogether with iron supplement. She had no more abdominal discomfort at 2 weeks after the medical treatment. Six weeks later, the patient underwent jejunal biopsies which showed unremarkable histopathology and she was stable at 2 years followed-up without any recurrence of anemia.

Conclusion: Giardiasis is a rare etiology of obscure overt gastrointestinal bleeding.

\section{IP1-7 \\ Enteroscopy Findings: Hookworm Infection As Cause of Severe Anemia}

Amanda Pitarini Utari, Hasan Maulahela, Ari Fahrial Syam, Murdani Abdullah, Dadang Makmun

Department of Internal Medicine, Faculty of Medicine, Universitas Indonesia, Indonesia

Enteroscopy is one of the tests to diagnose small bowel bleeding. Most frequent indications for enteroscopy in our endoscopy center are obscure GI bleeding, followed by abdominal pain. During 20122016, etiology of small bowel bleeding found during enteroscopy includes small bowel tumor, ulcerations, vascular malformation, and NSAID enteropathy.

We presented two cases of female farmers, age 52 and 67 year, who were referred to our hospital for severe anemia (hemoglobin level were $3.5 \mathrm{~g} / \mathrm{dL}$ and $4.6 \mathrm{~g} / \mathrm{dL}$ ). Upper GI endoscopy in both patient only found mild hyperemia in antral region. Colonoscopy was normal in one patient and melena in another patient. We decided to perform enteroscopy in both patient, and found hook worms in the duodenum and jejunum. Hookworm infection is prevalent in tropical and subtropical country. A study in rural area in Indonesia reported that $92.2 \%$ of participant were infected with at least one species of helminth. About $53.5 \%$ of them was hookworms.

Hook worm infection is still one of possible etiology of severe anemia in middle-age patient in Indonesia.

\section{IGICS Poster Session 2}

\author{
Chairperson: Ki-Baik Hahm (CHA University School of \\ Medicine, Korea)
}

\section{IP2-1 \\ Comparison of Mucosal Defect Size and Resection Depth After Hot and Cold Snare Polypectomy}

Sho Suzuki, Takuji Gotoda, Chika Kusano,
Hisatomo Ikehara, Hiroaki Okuno, Kaori Hayashi,
Mitsuru Esaki, Mitsuhiko Moriyama

Division of Gastroenterology and Hepatology, Department of Medicine, Nihon University School of Medicine

Background and Aims: Hot snare polypectomy (HSP) and cold snare polypectomy (CSP) are commonly used for treatment of small colorectal polyps. The resection range achieved with 2 methods are still unknown. This study compared the mucosal defect size and resection depth achieved with HSP or CSP for small colorectal polyps.

Methods: This was a single-center, prospective, randomized controlled study. Fifty-two patients with 56 rectal or rectosigmoid polyps up to $10 \mathrm{~mm}$ in diameter were enrolled in this study. The patients were randomized for treatment with either HSP or CSP.
Digestion 2019;99:95-119

DOI: $10.1159 / 000494404$
The 11th International Gastrointestinal Consensus Symposium (IGICS) 
Mucosal defect size after each procedure was measured accurately using an endoscopic measuring device immediately after the procedure and 1 day later. Resection depth was histologically evaluated using specimens obtained from each procedure.

Results: The mean size of the lesions treated with HSP $(\mathrm{n}=28)$ and CSP $(\mathrm{n}=28)$ was $5.6 \mathrm{~mm}$ and $5.6 \mathrm{~mm}$, respectively $(\mathrm{P}=0.938)$. The mean diameter of the mucosal defect 1 day after HSP and CSP was $6.3 \mathrm{~mm}$ and $5.4 \mathrm{~mm}$, respectively $(\mathrm{P}=0.056)$. The diameter of the mucosal defect 1 day after the procedure increased by $28.1 \%$ with HSP and decreased by $24.5 \%$ with CSP $(P=0.001)$. Submucosal tissue was obtained significantly more often with HSP than with CSP $(82.1 \%$ [23/28] vs. $28.6 \%$ [8/28], $\mathrm{P}=0.001)$. The mean thickness of submucosal tissue from the muscularis mucosae to the resection margin was significantly greater in specimens obtained with HSP than in those obtained with CSP $(0.939 \mathrm{~mm}$ vs. $0.064 \mathrm{~mm}, \mathrm{P}=0.001)$.

Conclusion: Mucosal defects increased in size within 1 day of HSP; however, they decreased within 1 day of CSP. The resection depth in HSP was deeper than that in CSP. These findings could account for the higher curability and higher risk of adverse events after HSP.

\section{IP2-2}

\section{Linked Color Imaging (LCI) is Clinically Useful for Endoscopic Assessment in Ulcerative Colitis: A Preliminary Pilot Study with 10 Patients}

\author{
Kohei Yamanouchi', Ryuichi Iwakiri', Ryo Shimoda", \\ Yasuhisa Sakata², Nanae Tsuruoka², Kazuma Fujimoto ${ }^{1,2}$ \\ ${ }^{1}$ Department of Internal Medicine \& Gastrointestinal \\ Endoscopy, Takagi hospital, Japan; ${ }^{2}$ Department of \\ Internal Medicine, Saga Medical School, Japan
}

Background and Study Aims: Linked color imaging (LCI) is a novel image-enhanced endoscopy technique which increases distinctiveness of the mucosal color of endoscopic images. The aim of this study is to investigate the usefulness of LCI to evaluate the activity of mucosal inflammation in ulcerative colitis (UC) patients.

Patients and Methods: Totally, 10 consecutive patients with inactive or mildly active UC were enrolled, and fifty-three areas were assessed by LCI. All examinations were conducted with a LASEREO endoscopic system (FUJIFILM CO., Tokyo, Japan). During the colonoscopy, each region of interest (ROI) was observed by both white light imaging (WLI), and LCI. The Commission international de l'éclairage (CIE) LAB color diferences (E) were calculated among WLI and LCI in each ROI. It is considered that the difference of color can be recognized if $\mathrm{tE}$ is more than 13 . Therefore, recognition of color difference was classified as; easy recognition of color difference, $\mathrm{E}>13$; difcult recognition of color difference, $\mathrm{E}<13$. After observed by colonoscopy, the biopsy specimen was taken in each ROI. The correlation between $\mathrm{hE}$ and each histological finding were assessed, and between $\mathrm{E}$ and Mayo endoscopic sub-score were assessed too.

Results: In the multivariate analysis, color difference of ROI without inflammatory cell infiltration could be recognized significantly more easily than that of ROI with inflammatory cell infiltration by using LCI (OR: 6.8, 95\% CI: 1.26-36.81, $\mathrm{p}=0.03$ ). Recognition of color difference was not affected by the other histological find- ings. As a result of analyzing the color tone, LCI distinguished colon mucosal white color compared to WLI, indicating the remissioncolon mucosa of UC with no inflammatory cell infiltration in ROI was easily detected by LCI. In the case of Mayo endoscopic subscore was 0 , color difference could be recognized significantly more easily compared to Mayo endoscopic sub-score was 1-3 (OR:5.7, 95\% CI:1.31-24.47, $\mathrm{p}=0.02$ ).

Conclusions: The inactive UC mucosa could be easily detected as the white area by the LCI mode compared to WLI, suggesting that LCI might be one of novel approaches for evaluation of disease activity of UC.

\section{IP2-3 \\ Pretreatment of Probiotic Cocktail Bifico Ameliorates Colitis-Associated Colorectal Cancer in Mice: Transcriptomic Analysis and Gut Flora Profiling}

\section{Huan Song}

Department of Gastroenterology, Ruijin Hospital, Shanghai Jiaotong University School of Medicine, China

Background: Individuals with inflammatory bowel disease (IBD) are at a high risk for developing colitis-associated colorectal cancer (CAC). Strategies to block the process from IBD to CAC should be considered. In the present experiment, we aim to explore the chemopreventive efficacy of the probiotic cocktail Bifco and its potential mechanism in azoxymethane (AOM) and dextran sodium sulphate (DSS) induced colitis-associated colorectal cancer.

Methods: Pretreatment of Bifco in mice with CAC was adopted to evaluate the protective effect of this probiotic mixture. The colorectums of mice were collected and examined for inflammatory degree and presence of tumors. Methods of cDNA microarray, comparative 16S rRNA sequencing and following bioinformatic approach were performed to observe Bifico-target as well as AOM/DSS-induced alterations in gene expression profile and microbiota community.

Results: Pretreatment of Bifco significantly reduced the CAC tumor formation and alleviated intestinal inflammation. Furthermore, we acquired transcriptomic changes and microbiota structure alterations in response to Bifico-treatment in AOM/DSS-induced CAC mouse model and identified a subset of genes as potential targets involved in the preventive effect of Bifico treatment, including chemokine $\mathrm{C}-\mathrm{X}-\mathrm{C}$ motif ligand 1 (CXCL1), CXCL2, CXCL3, and CXCL5, which were all ligands of $\mathrm{C}-\mathrm{X}-\mathrm{C}$ motif receptor 2 (CXCR2). 16S rRNA sequencing demonstrated that Bifico-treatment decreased the abundance of genus Desulfovibrio, Mucispirillum and Odoribacter, while a bloom of genus Lactobacillus was detected in the mucosa-attached microbiota. Notably, we found that abundance of these Bifico-target taxa was associated with the mRNA expression of CXCR2 ligand genes.

Conclusions: Our studies demonstrate that oral administration of Bifico ameliorate colitis-associated colorectal cancer in mice through intervening with the possible mechanistic link between Desulfovibrio, Mucispirillum, Odoribacter, Lactobacillus and CXCR2 signaling pathway. Moreover, frameworks of changes in gene expression profile and gut flora community caused by Bifico in AOM/DSS-induced CAC mouse model and AOM/DSS alone have been established. 


\section{IP2-4 \\ The Association of Colonic Diverticular Disease and Colonic Adenoma in Filipinos}

Clarel Camille B. Ng, Maria Carla V. Tablante, Jose D. Sollano

University of Santo Tomas, Manila, Philippines

Background and Objective: The prevalence of both colonic diverticulosis and adenoma is high in the elderly. Reports from the west comprise the majority of the evidence and thus, it is the objective of this study to determine the association of colonic adenomas with diverticular disease in a Filipino cohort.

Method: From 2014 to 2016, all adult patients who had a complete colonoscopy in a tertiary teaching hospital were recruited. Demographic data including age, gender, BMI, smoking status and comorbidities of patients with diverticulosis and adenoma (Group 1) were compared to those with adenoma but without diverticulosis (Group 2). Withdrawal time and use of sedation were also recorded. Data analyses were determined using chi square, logistic regression analysis and the student $t$ test.

Result: A total 2736 patients met the inclusions criteria; 651 $(23.8 \%)$ have diverticulosis and $658(24 \%)$ have adenomas. Colonic adenomas are found in $30.7 \%$ and $22 \%$ of patients with (Group 1) and without diverticulosis (Group 2), respectively [ (OR 1.6 vs 0.625 ; CI 1.3-1.9), $\mathrm{p}=0.001]$. Patients are significantly older in Group 1 than in Group $2(65.6 \pm 11$ and $59.1 \pm 13$ years, $p$ value $<0.001)$. The male to female ratio is $1.7: 1$ ( $\mathrm{p}$ value 0.938 ). The distribution of adenomas is not significantly different in left-sided, right-sided and diffused diverticular disease, i.e., $40.5 \%, 36 \%$ and $23.5 \%, \mathrm{p}=0.519$, respectively. Hypertension (OR 1.42; CI 1.02-1.99) and diabetes mellitus (OR 2.29; CI 1.59-3.3) increase the likelihood of finding an adenoma in patients with diverticulosis. Scope withdrawal time $>6$ minutes and deep sedation both yielded higher adenoma detection but not significantly different between both groups ( $\mathrm{p}$ value $=0.387$ and 0.481 , respectively).

Conclusion: Diverticulosis increased the likelihood of finding adenomas during colonoscopy. The predictive factors for finding adenomas in patients with diverticulosis are age $>60$ years, hypertension and diabetes mellitus.

\section{IP2-5}

\section{Diverticular Disease of the Colon: An Analysis of the Clinical Characteristics and Risk Factors in Adult Filipino Patients}

\author{
Clarel Camille B. Ng, Maria Carla V. Tablante, \\ Jose D. Sollano \\ University of Santo Tomas, Manila, Philippines
}

Background and Aim: Colonic diverticular disease is a common finding in adults undergoing colonoscopy. There are only few reports on the characteristics of diverticulosis in the Asian population thus, we aim to describe the clinical characteristics and risk factors of Filipino patients with colonic diverticular disease.
Method: All patients undergoing colonoscopy from January 2014 to December 2016 in our center were enrolled into the study. From a total of 3480 patients, we included only 2736 adult patients who had a complete colonoscopy. The number and location of colonic diverticular were analyzed and correlated with age, BMI, smoking status, co-morbidities, withdrawal time and sedation status.

Result: The prevalence of diverticulosis is $23.8 \%(651 / 2736)$. It is more prevalent in males $(62 \% 403 / 651)$ and the mean age of patients diagnosed with diverticulosis is $62 \pm 12$ years old. Majority of the colonoscopies were done for hematochezia (30\%) in males and, hematochezia (27.8\%) and abdominal pain (27.8\%) in females. Most of the diverticula are located in the right colon $(38.6 \%, 251 / 651)$. The prevalence of pancolonic diverticulosis significantly increases with advancing age. In our study, there is no association between BMI and colonic diverticulosis. Factors predictive for the presence of diverticulosis include age $>50$ years (OR 2.58; CI 1.99-3.34), male gender (OR 1.2; CI 1.08-1.6), smoking (OR 1.6; CI 1.3-2.0), hypertension (OR 1.7; CI 1.45-2.1), diabetes (OR 2.4; CI 2.0-3.0), atherosclerosis (OR 2.5; CI 1.74-3.71), dyslipidemia (OR 1.54; CI 1.22-1.95) and metabolic syndrome (OR 1.1; CI 0.69-1.75). Mean withdrawal time (13.30 minutes \pm 9.8 ) and type of sedation administered do not influence the diagnosis of diverticulosis.

Conclusion: Up to a quarter of adult Filipino patients have diverticulosis which are predominantly located in the right colon. The most common clinical presentation is hematochezia and abdominal pain, Male gender, smoking hypertension, diabetes, dyslipidemia and the metabolic syndrome increase the likelihood of diverticulosis in our population.

\section{IP2-6}

\section{Retrospective Study of the Preoperative Chemical Disinfection Methods; Olanexidine Gluconate vs. Conventional Method in Colorectal Surgery}

\author{
Yoshihiro Shirai, Naotake Funamizu, Katsushi Dairaku, \\ Yuki Hiramoto, Kenta Tomori, Tomonori lida, \\ Yukio Nakabayashi
}

Department of Gastrointestinal Surgery, Kawaguchi Municipal Medical Center, Japan

Introduction: Surgical site infection (SSI) is one of the major postoperative complication in colorectal cancer. Olanexidine gluconate is a new anti-septic solution approved by Japan in Sep 2015. We compared the incidence of SSI between olanexidine gluconate and popidone iodine.

Methods: We used popidone iodine $(\mathrm{n}=40)$ from July 2016 to December 2016 for open colorectal surgery for malignancies in Kawaguchi Municipal Medical Center excluding of an emergency surgery. Olanedine $(n=40)$ was used from January to June 2017. We compared to the incidence of SSI between two periods. SSI was defined according Center for Disease Control and Prevention (CDC) criteria. The incidence of SSI was $20 \%$ in all patients. The respective incidence of SSI were $20 \%$ in olanedine gluconate and $20 \%$ in popidone iodine $(\mathrm{p}=1.000)$. There were no significant differences in both groups with age $(\mathrm{p}=0.748)$, gender $(\mathrm{p}=0.805)$, surgical time
116

Digestion 2019;99:95-119

DOI: $10.1159 / 000494404$
The 11th International Gastrointestinal Consensus Symposium (IGICS) 
$(p=0.522)$, types of cancer $(p=0.152)$, ASA score $(p=0.369)$ and colostomy $(\mathrm{p}=0.104)$.

Conclusion: Olanexidine gluconate seems to be a novel and an useful anti-septic solution without complication.

\section{IP2-7 \\ DNA Methylation Biomarker in Biliary Brush Sample for Diagnosis of Cholangiocarcinoma in Patients with Bile Duct Stricture}

\author{
Papatsakorn Nopjaroonsri ${ }^{1}$, Peti Thuwajit ${ }^{2}$, \\ Udom Kachintorn", Varayu Prachayaku/ ${ }^{1}$ \\ 'Division of Gastroenterology, Department of Medicine, \\ Siriraj Hospital, Mahidol University, Thailand; ${ }^{2}$ Department \\ of Immunology, Siriraj Hospital, Mahidol University, \\ Thailand
}

Introduction: Extrahepatic cholangiocarcinoma (ECC) usually manifests as obstructive jaundice which is not very easy to distinguish between this fatal bile duct malignancy and other benign conditions. Endoscopic Retrograde Cholangiopancreatography (ERCP) with brushed cytology is still a standard method for diagnosis of ECC, however, it give a low diagnostic yield despite high specificity but less sensitivity. Thus, to improve the diagnostic sensitivity, molecular study was introduced. Recently, based on our unpublished previous pilot study, the hypermethylation status of single or combination of HOXA1 and NEUROG1 genes was found statistically significantly higher in patients with ECC than normal biliary epithelium. The aim of this study is to evaluate these biomarkers panel for improving diagnosis of ECC by DNA methylation analyses of abnormal bile duct epithelial cell from biliary brush sample in the patients with biliary stricture.

Methods: Biliary brush samples from 24 patients with biliary stricture (14 ECC and 10 benign conditions for control group) who underwent ERCP were examined for methylation status of HOXA1 and NEUROG1 genes using quantitative methylation-specific polymerase chain reaction. Receiver operating characteristic curve analyses were used to evaluate the performance of individual biomarkers. The study design is a Single center, cross-sectional study.

Result: The methylation NEUROG1 gene was investigated in 24 biliary brush samples (14 ECC and 10 benign conditions for controls). NEUROG1 gene achieved 13/14 (92.9) and 1/10 (10.0) for ECC and benign biliary stricture $(\mathrm{p}<0.01)$ with $92.9,90$ and $91.7 \%$ sensitivity, specificity and accuracy respectively under an area under the receiver operating characteristic curve of 0.907 . Cytology achieved 12/14 (85.7) for ECC with 85.7, 100 and $83.3 \%$ sensitivity, specificity and accuracy respectively. The combined sensitivity of NEUROG1 gene and cytology is $100 \%$ for diagnosis of ECC.

Conclusion: Hypermethylation of NEUROGl gene is benefit and could improve diagnostic sensitivity of routine cytology for extrahepatic cholangiocarcinoma. 
Abdullah, M. IP1-1, IP1-7

Adachi, T. IO4-2

Akashi, T. IO3-1

Akazawa, Y. IO3-1, IO4-6

Amagase, K. IO4-3

Ando, Y. IP1-4

Aoki, Y. IO3-6

Asaoka, D. IO4-6

Aswakull, P. IP1-6

Aziza, S. IP1-1

Baba, H. IO1-2

Bang, B.W. IO4-4

Cai, C. IO4-5

Cao, L. IO3-5

Chan, F.K.-L. IO1-8

Chan, F.K.L. IO2-3

Chan, H.L.Y. IO1-8

Chen, C. IO4-5

Chen, Z. IO3-5

Cho, I.K. IO3-3

Cho, J.Y. IO1-3, IP1-3, IO3-4, IO4-1

Coker, O.O. IO3-5

Dai, Z. IO3-5, IO4-5

Dairaku, K. IP2-6

Esaki, M. IO2-6, IP2-1

Fan, Y. IO4-5

Fauzi, A. IO1-7

Fernandez, D.F. IO2-5

Fock, K.M. IO2-1

Fujikawa, Y. IO1-5

Fujimoto, K. IP2-2

Fujiwara, M. IO2-6

Fukumoto, M. IO1-5

Funamizu, N. IP2-6

Funasaka, K. IP1-4

Furukawa, K. IP1-4

Goto, H. IP1-4

Gotoda, T. IP2-1

Hahm, K.B. IO1-3

Hahm, K.-B. IP1-2, IP1-3, IO3-3

Hahm, K.B. IO3-4

Hahm, K.-B. IO4-1

Hahn, K.Y. IO3-2
Hamouda, N. IO4-3

Han, Y.M. IO1-3

Han, Y.-M. IP1-2, IP1-3, IO3-3

Han, Y.M. IO3-4

Han, Y.-M. IO4-1

Harada, S. IO1-5

Hayashi, K. IP2-1

Higuchi, K. IO1-5, IO4-3

Hiki, N. IO1-2

Hiramoto, Y. IP2-6

Hirata, Y. IO2-4

Hirooka, Y. IP1-4

Hojo, M. IO4-6

Hong, S.P. IO1-3, IP1-2, IP1-3, IO3-3, IO3-4, IO4-1

Hosoya, A. IO4-2

$\mathrm{Hu}, \mathrm{N}$. IO4-5

Huang, M. IO4-5

Iida, T. IP2-6

Ikehara, H. IP2-1

Imamura, Y. IO1-2

Isomoto, H. IO1-1, IO3-1

Itoi, T. IO3-6

Iwakiri, R. IP2-2

Izumi, K. IO4-6

Janyangdikul, P. IP1-6

Joh, T. IO2-4

Kachintorn, U. IP1-6, IP2-7

Kamiya, M. IO3-1

Kanda, T. IO1-1, IO3-1

Kang, E.-A. IP1-3, IO3-3

Kang, E.A. IO3-4

Kang, Y. IP1-2, IP1-3, IO3-3, IO4-1

Kato, S. IO4-3

Kawai, T. IO3-6

Kawano, S. IO2-6

Kawasaki, K. IO2-6

Kim, D.H. IO4-1

Kim, N. IO2-2

Kim, S.B. IO2-2

Kim, Y.K. IO2-2

Ko, W.J. IO1-3, IO3-4

Kojima, Y. IO1-5, IO4-2

Komori, H. IO4-6

Koyama, Y. IO3-6

Kurumi, H. IO1-1

Kusano, C. IP2-1
Lai, L. IO4-5

Lee, D.H. IO2-2

Lee, D.-Y. IP1-2

Lee, H.J. IO2-2

Lee, N.K. IO3-2

Lee, S.J. IO2-2

Lee, S.K. IO3-2

Li, J.W. IO2-1

Liang, J.Q. IO2-3

Madarame, A. IO3-6

Maeda, O. IP1-4

Maehara, Y. IO1-2

Makmun, D. IP1-1, IP1-7

Matsumoto, K. IO4-3, IO4-6

Matsumoto, T. IO2-6

Matsuno, Y. IO2-6

Matsuoka, A. IP1-4

Matsushima, K. IO3-1

Maulahela, H. IO1-7, IP1-7

Maulahela, S. IP1-1

Mine, S. IO1-2

Miyahara, R. IP1-4

Morise, T. IO3-6

Morita, M. IO1-2

Moriyama, M. IP2-1

Nagahara, A. IO4-6

Nagaishi, T. IO4-2

Nakabayashi, Y. IP2-6

Nakamura, S. IO2-6

Nakano, M. IO3-1

Nakao, K. IO3-1

Nakatsu, G. IO3-5

Nakazawa, T. IO2-4

Ng, C.B. IO2-5

$\mathrm{Ng}$, C.C.B. IP2-4, IP2-5

Nie, Y. IO3-5

Nisha, J. IO4-2

Nopjaroonsri, P. IP2-7

Nursyirwan, S.A. IP1-5

Ogiwara, K. IO1-1

Oh, J.-Y. IP1-2

Ohnita, K. IO3-1

Oki, E. IO1-2

Okuda, Y. IO2-4

Okuno, H. IP2-1

Ota, K. IO1-5

Ozaki, H. IO1-5

Park, J.M. IO1-3
Park, J.-M. IP1-2, IP1-3, IO3-3

Park, J.M. IO3-4

Park, J.-M. IO4-1

Park, S.H. IO2-2

Park, Y.S. IO2-2

Phoa, T.B. IO2-5

Prachayakul, V. IP1-6, IP2-7

Qiao, Y. IO4-5

Ran, Z. IO4-5

Rani, A. IO1-7

Rani, A.A. IP1-1

Sakamoto, E. IO2-4

Sakata, Y. IP2-2

Sano, T. IO1-2, IO4-3

Seo, A.Y. IO2-2

Shen, J. IO4-5

Shimada, Y. IO4-6

Shimakawa, M. IO4-3

Shimoda, R. IP2-2

Shimura, T. IO2-4

Shin, C.M. IO2-2

Shin, S.A. IO3-4

Shin, Y.W. IO4-4

Shirai, Y. IP2-6

Sofue, S. IO2-4

Sollano, J.D. IP2-4, IP2-5

Son, W.C. IO2-2

Song, D. IO4-5

Song, H. IP2-3

Sugimoto, A. IO3-6

Sun, Y. IO4-5

Sung, J.J.Y. IO2-3, IO3-5

Suzuki, S. IP2-1

Syam, A.F. IP1-5, IP1-7

Tablante, M.C.V. IP2-4, IP2-5

Takeda, T. IO4-6

Takeshima, F. IO3-1

Takeuchi, T. IO1-5

Thuwajit, P. IP2-7

Tiing, L.A. IO1-6

Tominaga, K. IO1-5

Tomori, K. IP2-6

Torisu, T. IO2-6

Tse, Y.K. IO1-8

Tsuji, Y. IO3-6

Tsuruoka, N. IP2-2

Uchida, K. IO3-6 
Ueda, Y. IO1-5

Ueyama, H. IO4-6

Urano, Y. IO3-1

Utari, A.P. IO1-7, IP1-7

Wang, C. IO4-5

Wang, D. IO1-4

Wang, T. IO4-5

Watabe, T. IO4-2
Watanabe, M. IO1-2, IO4-2

Watanabe, S. IO4-6

Wong, G.L.H. IO1-8

Wong, S.H. IO3-5

Wong, V.W.S. IO1-8

Wu, W.K.K. IO3-5

Yagi, K. IO3-6

Yamada, T. IO2-4
Yamaguchi, R. IO2-4

Yamamoto, M. IO1-2

Yamanouchi, K. IP2-2

Yanai, S. IO2-6

Yip, T.C.F. IO1-8

Yoo, I.K. IP1-2, IP1-3

Yoon, H. IO2-2

Youn, J.-H. IO3-2

$\mathrm{Yu}, \mathrm{J}$. IO2-3, IO3-5
Zhao, D. IO4-5

Zhao, H. IO3-5

Zheng, Q. IO4-5

Zhou, M. IO4-5

Zhu, M. IO4-5 